\title{
I PARTITI CONSERVATORI IN EUROPA. UN SUCCESSO DA SPIEGARE
}

di Francesco Raniolo

Premessa

Alla fine degli anni '80 Girvin affermava che in quel decennio c'era stato un movimento tanto nelle politiche che nella politica verso la destra $(1988,4)$ il cui aspetto immediatamente visibile in Europa era stato il marcato predominio conservatore sul piano elettorale. Due date possono essere assunte come punti di riferimento: le elezioni britanniche del 1979 che consentirono al Partito conservatore, sotto la guida della Thatcher, di andare al governo e di restarvi per i successivi diciotto anni e, negli Stati Uniti, l'elezione di Reagan nel 1980 e nel 1984.

Etichette, assai popolari negli anni ' 80 , come reaganismo e thatcherismo stavano a testimoniare l'affermazione di un nuovo modello di «consenso neo-conservatore» o «neo-liberale» che avrebbe finito con il dare un'impronta inusitata alle policies e ai politics changes delle liberaldemocrazie occidentali. «In larga parte questi cambiamenti rappresentarono una rivoluzione nelle «idee» che è meglio resa nei termini del concetto di policy paradigms [...]» (Hall 1992, 91), cioè un cambiamento a livello, per così dire, della Gestalt che «strutturava i diversi modi attraverso i quali i policy-makers vedevano il mondo e il loro ruolo all'interno di esso» (ibidem, 92).

Come cercheremo di precisare nel corso dell'articolo, i principali beneficiari «dell'ondata di destra» - boyrebolge o böger-

Questo saggio costituisce un'ampia rielaborazione delle conclusioni della mia tesi di dottorato («I partiti conservatori nell'Europa Occidentale. Andamenti elettorali, memberships e partecipazione al governo», 1997). Un particolare ringraziamento va al prof. Leonardo Morlino per $i$ consigli e suggerimenti che mi ba fornito nel corso dell'attività di ricerca e nella stesura di questo saggio. Cosi come debbo ringraziare il lettore anonimo della RISP per le sue preziose osservazioni. Tutta mia è comunque la responsabilità per quanto qui sostenuto.

RIVISTA ITALIANA DI SCIENZA POLITICA / a. XXVIII, n. 2, agosto 1998 
vag (Ljunggren 1988, 126), per usare un'espressione diffusa in Scandinavia - sono stati proprio i partiti appartenenti alla «famiglia conservatrice».

Infatti, senza voler anticipare aspetti che verranno affrontati con più attenzione nei successivi paragrafi, basti dire che dal confronto tra gli andamenti elettorali dei partiti di destra risalta la prevalenza delle formazioni politiche conservatrici di ispirazione laica ${ }^{1}$. In particolare, sotto il profilo dei rapporti di forza tra le varie componenti interne alla destra europea (la dimensione intra-blocco), emerge una sostanziale incompatibilità di famiglia ideologica o, per usare un'espressione di Lane e Ersson (1996), una «idiosincrasia partitica» tra forze politiche di ispirazione liberal-conservatrice e confessionale.

Sul piano ideologico-programmatico, e siamo ad un secondo motivo di interesse, $i$ partiti liberal-conservatori rappresentano la più pura incarnazione delle issues politiche tipicamente di destra, o meglio della cosiddetta «nuova-destra», diffuse in questo scorcio di secolo ${ }^{2}$. Com'è stato ricordato, in Europa sia i conservatori che i non-conservatori hanno finito per abbracciare l'ideologia «conservatrice» del libero mercato [sic!] (Ware 1996, 26).

Una terza ragione di interesse attiene al fatto che, nonostante la rilevanza dell'argomento, ai partiti conservatori è stata finora dedicata scarsa attenzione in sede comparata. Negli ultimi quindici anni, mentre non sono mancati lavori pregevoli su singoli partiti (specialmente per i Conservatori britannici e i Gollisti francesi), l'analisi comparata della famiglia conservatrice è stata sostanzialmente trascurata rispetto, per esempio, al più re-

1 Come vedremo nel prossimo paragrafo per classificare i partiti europei prenderemo come punto di partenza la tipologia di Beyme (1987). In particolare, ne metteremo in risalto le quattro famiglie ideologiche accomunate dal posizionamento a destra del centro: quella democristiana (o confessionale), quella dei liberali, quella dei conservatori (o dei liberal-conservatori) e, infine, quella delle formazioni di estrema destra. Per completezza del quadro comparativo indicheremo anche $\mathrm{i}$ cosiddetti partiti rurali caratteristici dei sistemi politici del nord Europa.

$2 \mathrm{Nel}$ corso di questo lavoro l'espressione «nuova destra» verrà utilizzata come sinonimo di «neoliberalismo», ad indicare «quei conservatori che si schierano a favore di un'illimitata espansione delle forze di mercato» (Giddens 1997, 34). Ora, se è corretta in Europa, questa scelta terminologica è invece fuorviante se riferita all'esperienza degli Stati Uniti. «Negli Stati Uniti [nuova destra e neoliberalismo] non assumono lo stesso significato che viene loro attribuito in Europa. La nuova destra americana tende ad essere associata alla destra religiosa protestante. I "liberali" degli Stati Uniti non coincidono con i liberali di Manchester: sia nel periodo del New Deal sia successivamente, essi hanno combattuto, in realtà, per l'espansione del welfare state» (Giddens 1997, 34). 
cente interesse per i partiti di estrema destra (Beyme 1987; Ignazi e Ysmal 1992; Ignazi 1994). Più in generale, ai partiti moderati e conservatori sono stati dedicati pochi lavori. Tra i più noti si possono ricordare: quelli sui partiti democristiani di Irving (1979) e, più di recente, di Caciagli et al. (1992); i due volumi sui partiti liberali di Bogdanor (1983) e di Kirchner (1988); sui partiti conservatori, invece, abbiamo il volume dei primi anni '80 di Leyton-Henry (1982) e la già ricordata antologia curata da Girvin (1988), ai quali si può aggiungere il lavoro di Morgan e Silvestri (1983).

Infine, una quarta ragione per puntare l'attenzione sui partiti conservatori deriva dalle stesse vicende che hanno caratterizzato il caso italiano dopo le elezioni del marzo 1994. In sostanza, il passaggio alla cosiddetta Seconda Repubblica (per altro tuttora in corso) per tutta una serie di aspetti significativi - la diaspora democristiana, la riarticolazione del sistema partito attorno a due poli, la fulminea affermazione di Forza Italia e lo stesso spostamento nell'orbita moderata e di governo di Alleanza nazionale - sembrerebbe avviare il sistema politico italiano verso una nuova stagione dei rapporti tra confessionalismo e conservatorismo politico. Il che, almeno a sentire alcuni protagonisti, dovrebbe avere come esito finale proprio la costruzione di un moderno partito liberal-conservatore di stampo europeo da qui anche importanti spunti per la comparazione con la Francia della Quinta Repubblica e con le altre democrazie del Sud Europa.

Alla questione della classificazione dei partiti di destra delle sedici democrazie dell'Europa occidentale qui prese in esame sarà dedicato il prossimo paragrafo. I tre paragrafi successivi saranno, invece, dedicati all'analisi comparata delle tre principali dimensioni della loro azione partitica - i risultati elettorali, gli andamenti delle iscrizioni e la permanenza al governo. Il confronto sistematico dei partiti conservatori con le altre formazioni politiche, e non solo di destra, ci permetterà di tracciare un quadro delle performances e della salienza che essi hanno nei diversi sistemi politici. Nelle conclusioni, infine, proporremo due ipotesi interpretative, complementari, del «successo» di questi partiti: l'ipotesi dell'ambiente generale di riferimento, e l'ipotesi del vantaggio competitivo. 


\section{Che cosa comparare}

La migliore strada per affrontare le questioni sollevate nelle pagine precedenti, senza correre il rischio di incappare in qualche «superstizione politologica» (Panebianco 1986), è data dal ricorso alla comparazione. Una volta individuato l'oggetto e l'ambito della ricerca, il primo passo richiesto dalla comparazione è di natura classificatoria (Dogan e Pelassy 1990; Sartori e Morlino 1991). Nel nostro caso si tratta, innanzitutto, di indicare quali partiti dell'Europa occidentale considerare di destra e, in particolare, quali etichettare come conservatori ${ }^{3}$. Questa operazione apparentemente semplice presuppone la definizione dei criteri idonei a collocare i singoli casi empirici (i partiti) nelle rispettive classi. A tal scopo, abbiamo fatto ricorso a due dimensioni: quella storico-ideologica e quella sistemico-competitiva.

La scelta della dimensione storico-ideologica muove dalla constatazione che, in un certo senso, un partito è la sua storia (Ware 1996). «Differenti partiti è probabile che abbiano differenti interessi, o almeno una differente gerarchia di interessi, come risultato di differenti tradizioni ideologiche e di distinti impegni programmatici» (Budge e Keman 1990, 90). La classificazione dei partiti europei in termini di familles spirituelles o di «famiglie politico-ideologiche» ${ }^{4}$, cioè della loro adesione ad una specifica «comunità di valori e di norme» (Beyme 1987, 17), ci è sembrata la scelta migliore per tener conto del peso della loro identità politica sedimentata. Abbiamo, così, ripreso la proposta avanzata già agli inizi degli anni '80 da Beyme (1987, 11-14 e 19-123) e, più di recente, rilanciata da Ware $(1996,21-43)^{5}$.

La scelta del criterio delle «famiglie ideologiche» solleva, però, alcune questioni cruciali per il nostro ragionamento, a

3 In questa sede, invece, non è possibile affrontare una questione complementare a quella della classificazione dei partiti politici e relativa a che cosa debba intendersi per «destra» e per «conservazione» o, specularmente, per «sinistra» e per «progresso».

$4 \mathrm{La}$ distinzione tra famiglie politico-ideologiche non rinvia esclusivamente ad aspetti, per così dire, «spirituali» ma ha, anche, un preciso collegamento con $\mathrm{i}$ «grandi conflitti sociali che hanno determinato la nascita di determinati partiti» (Beyme 1987, 11-13), il che apre la possibilità di integrare l'approccio delle famiglie ideologiche con quello dei cleavages (Rokkan 1982). Inoltre, una considerazione più realistica dello schema delle famiglie politiche non deve sottovalutare l'avvertenza di Barnes $(1966,523)$ : «Ogni volta che le ideologie appaiono importanti in politica, ciò avviene perché possiedono una solida base organizzativa».

5 L'espressione «famiglia politica» deriva dalle familles spirituelles di Duverger (1961) e ha delle forti assonanze con le espressioni «tendenze di fondo» e «temperamento politico» introdotte nel 1913 nell'analisi elettorale da Siegfried. 
partire dal fatto che è alquanto difficile definire le varie famiglie partitiche, fissarne con certezza i confini e risolvere i problemi connessi alla classificazione dei casi marginali. C'è, poi, sempre il rischio di «stiracchiare» oltre il dovuto la capienza delle varie famiglie cercando di farvi rientrare quanti più partiti possibili. Sotto il profilo operativo, dunque, è bene muoversi in modo pragmatico, valutando caso per caso quali partiti inserire nelle famiglie previste dallo schema di Beyme ${ }^{6}$.

In secondo luogo, alcuni partiti di nuova formazione potrebbero sfuggire alla classificazione in termini di famiglie ideologiche tradizionali. In realtà, come si vede anche dagli esempi riportati in nota, il riferimento ai nuovi partiti è spesso sinonimo di «piccoli partiti» che si affermano, in genere, o per scissione da partiti più grandi $\mathrm{o}$, comunque, in funzione di protesta all'esterno dell'area di governo ${ }^{7}$. Per contro, la questione si fa più delicata quando le nuove formazioni politiche sono ben più rilevanti, sia per le dimensioni elettorali, sia per il ruolo istituzionale che ricoprono. Questo, per esempio, è quanto accade nelle transizioni di regime tanto «discontinue» (nel genere e nella specie) come quelle dell'Europa del sud, che «continue» (nel genere, comunque democratico, ma non nella specie) come nella Francia della Quinta Repubblica o nell'Italia dopo le elezioni del 1994.

Terza ed ultima osservazione: lo schema presentato nel testo deve essere visto in una prospettiva dinamica e non semplicemente statica, per cui «nessuna delle familles spirituelles do-

6 Secondo Ware $(1996,23)$, Beyme è incappato in uno di questi errori nel caso dell'Irlanda quando ha classificato il Fianna Fail nella famiglia dei conservatori e il suo principale avversario, il Fine Gael, in quella democratico-cristiana. Da parte nostra, comunque, abbiamo deciso di mantenere la collocazione dei due partiti fatta da Beyme (si veda anche Girvin 1988 e Budge 1995). Questa scelta, a fronte della sostanziale indistinzione ideologico-programmatica tra le due formazioni, poggia sulla loro stessa autodefinizione e auto-collocazione. Così, ad esempio, nell'ambito del Parlamento europeo, mentre il primo partito $(\mathrm{Ff})$ coerentemente con il suo nazionalismo e populismo è entrato a far parte del gruppo conservatore, il secondo $(\mathrm{Fg})$, invece ha aderito al gruppo democristiano (Jacobs 1989, 692 e ss.). Altri studiosi hanno proposto di classificare il Fine Gael nella famiglia confessionale e il Fianna Fäil, invece, nella famiglia liberale (Ersson e Lane 1987), per altro, riservando lo stesso trattamento al Partito socialdemocratico portoghese.

7 Si tratta comunque di evenienze che l'esistenza di uno stabile sistema partitico tende a neutralizzare, innanzitutto perché lo spazio politico è già occupato da partiti ben strutturati, poi per l'operare di vincoli istituzionali (a partire dal ruolo scoraggiante, rispetto ai nuovi ingressi, svolto dal sistema elettorale, comprendendo in esso la cosiddetta legislazione di contorno) e, infine, perché le scissioni possono essere penalizzanti in termini elettorali. 
vrebbe essere considerata come se fosse una categoria rigidamente demarcata nella quale ogni partito può essere collocato [una volta per tutte]» (Ware 1996, 24). Per svariate ragioni, i partiti nel corso del tempo possono cambiare la loro collocazione ideologica, orientarsi verso nuove alleanze, ridefinire le priorità e i temi della propria agenda programmatica ${ }^{8}$.

Queste considerazioni, se prese sul serio, spingono ad integrare il criterio storico-ideologico con quello sistemico-competitivo. Un'analisi che si fermi alla considerazione esclusiva dell'appartenenza ideologica corre il rischio di premiare delle affinità apparenti, delle uniformità esteriori (la collocazione nella stessa famiglia spirituale), trascurando così delle differenze sostanziali.

D'altra parte, un completo divorzio tra questi due aspetti per così dire, tra «teoria dichiarata» e «teoria in uso» dell'azione partitica - sarebbe del tutto irrealistico. Il giudizio comparativo sui partiti dovrebbe quindi tener conto dei loro comportamenti concreti, coalizionali o connessi agli orientamenti di policy. In generale, è opportuno correlare similitudini e differenze «al contesto in cui operano i partiti. Contesto, questo, che è largamente determinato dalla struttura del sistema partitico che fissa i vincoli e regola lo spazio competitivo» (Smith 1988, 20).

Del resto, i partiti politici non definiscono in isolamento i loro «contesti di controversia», le relative «immagini spaziali» e, più in generale, la loro stessa identità politica, ma, piuttosto, nell'ambito di un più ampio sistema di relazioni interpartitiche $\mathrm{e}$ di allineamenti partigiani (Sartori 1976; Bartolini e Mair 1990).

Abbiamo tenuto conto di queste precauzioni nella tabella 1 dove abbiamo classificato i partiti politici europei sulla base sia del criterio storico-ideologico - appartenenza, o almeno prossimità ideale, alle cinque famiglie ideologiche di Beyme (1987): confessionale, rurale, liberale, conservatrice e di estrema destra - sia del criterio spaziale-competitivo - collocazione dei partiti lungo la scala sinistra-destra (che varia da 0 a 10) di Castles e Mair (1984), i valori della quale sono indicati tra parentesi.

8 Con riferimento ai soli partiti di destra si possono ricordare il caso dell'Fpö austriaco, che a metà degli anni ' 90 si è sempre più spostato su posizioni radicali di destra o «populiste» (Taggart 1995; Ignazi 1994); dell'Ap/Pp spagnola che alla fine degli anni '70, specie dopo il risultato delle elezioni del 1982, ha progressivamente abbandonato le posizioni estremiste filo-franchiste (Caciagli 1993); e ancora del Msi/An che dopo le elezioni del 1994 sembrerebbe deciso (ma non senza tentennamenti) a chiudere i conti con la sua matrice fascista (Ignazi 1994). 
TAB. 1. Le famiglie ideologiche di destra nell'Europa occidentale

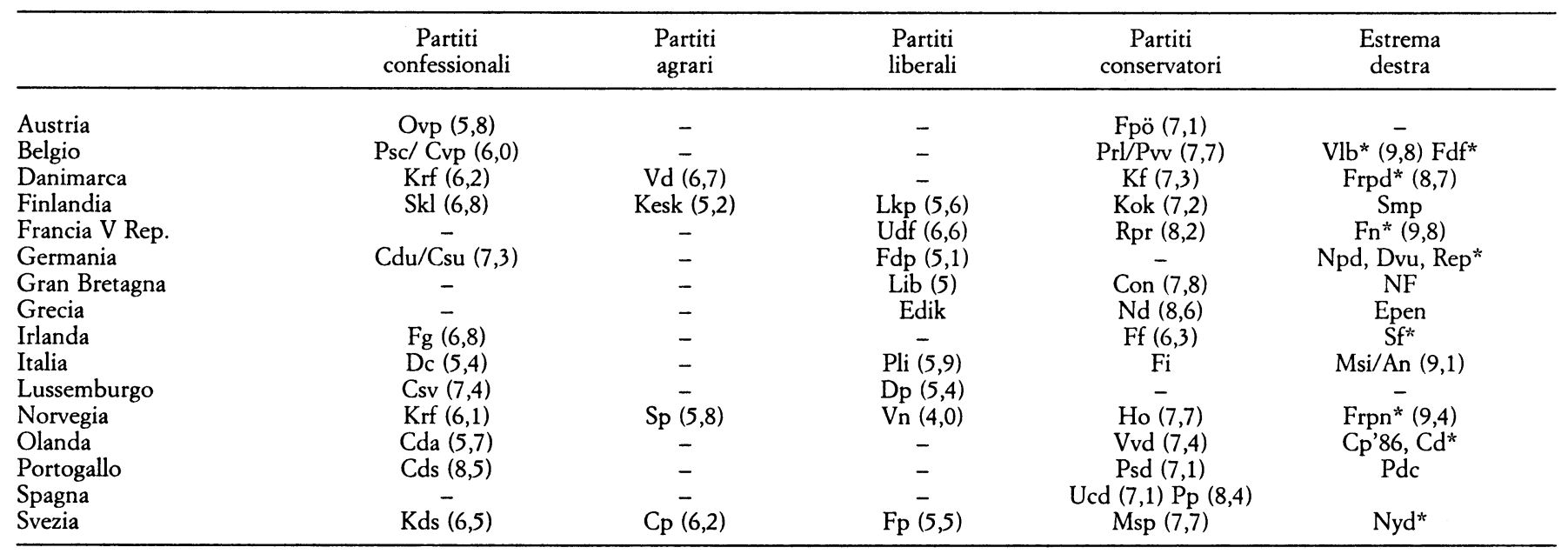

* Con l'asterisco sono indicati i partiti di estrema destra «nuovi», cioè la cui matrice storica ed ideologica non rinvia alla tradizione nazi-fascista (Ignazi 1994).

Fonti: i dati indicati tra parentesi, relativi alla collocazione dei partiti sulla scala sinistra-destra, si rifanno a Castles e Mair (1984), Humphreys e Steed (1988) e Colomer (1995). 
Da questa classificazione discendono alcune ben precise conseguenze operative. Innanzitutto, il riferimento al posizionamento dei partiti lungo l'asse sinistra-destra ci permette di distinguere tra partiti «moderati» (cioè con un punteggio compreso tra circa 6 e circa 8,5$)$ e partiti via via più «radicali» o estremisti (oltre 8,5). Per quanto riguarda questi ultimi, abbiamo preferito parlare genericamente di «estrema destra». Il riferimento spaziale in questo caso è piuttosto efficace nel discriminare. Sotto questa etichetta abbiamo annoverato un gruppo piuttosto composito per dimensioni elettorali, origini, organizzazione e matrice ideologica. Qui troviamo assieme tanto i partiti «vecchi» (Ignazi 1994), la cui ideologia rinvia cioè al nazi-fascismo, che i partiti «nuovi», «post-industriali» (ibidem) o «neopopulisti» (Taggart 1995), accomunati da una forte carica protestataria?.

Ma cosa dire di tutti quei partiti - conservatori, liberali e confessionali - che Castles e Mair $(1984,84)$ collocano nell'area della «destra moderata»? In particolare, ai fini del nostro ragionamento, il rapporto tra partiti liberali e partiti conservatori acquista un rilievo cruciale. Storicamente, questo rapporto è passato dall'aperto antagonismo alla sovrapposizione e, infine, (almeno per certi aspetti) alla fusione di valori, programmi e posizioni sulle politiche. In realtà, questa sovrapposizione non è stata mai perfetta. Da qui l'affermazione di due tipi di liberalismo, a seconda dell'enfasi data a particolari aspetti economici (laisserfaire o interventismo) e/o sociali (individualismo o comunitarismo): il «liberalismo-radicale», da un lato, e il «liberalismo-conservatore», dall'altro (Smith 1988, Humphreys e Steed 1988).

Queste osservazioni trovano conferma nella collocazione che i partiti liberali - o meglio, come si è visto, alcuni partiti liberali (tab. 1) - hanno sulla scala sinistra-destra. Infatti, come già avevano messo in evidenza Castles e Mair (1984) e Humphreys e Steed (1988), i partiti liberali austriaco (Fpö), belga $(\mathrm{Prl} / \mathrm{Pvv})$, olandese $(\mathrm{Vvd})$ e danese $(\mathrm{Vd})$, mostrano tutti una so-

9 Oramai è invalso l'uso di parlare a proposito dei partiti di estrema destra di «neo-populismo» o semplicemente di «populismo» (Lane e Ersson 1996), a volere indicare, oltre che un complesso di motivazioni ideologiche, tanto alcuni tratti sociologici (come, per esempio, il loro carattere «a-classista» più che «inter-classista») che organizzativi (ruolo predominante della leadership). L'uso di queste etichette, però, è stato criticato per il carattere fortemente contestualizzato di quelle espressioni. Infatti, esse rinviano, principalmente, ad alcune esperienze politiche tipiche dell'America Latina del XX secolo. 
vrapposizione spaziale con i partiti conservatori (il valore medio della collocazione lungo l'asse sinistra-destra è superiore ai 7 punti) e un comune profilo di policy ${ }^{10}$. Per questo motivo abbiamo deciso di riclassificarli, tutti tranne il partito liberale danese (Venstre), nella famiglia dei partiti conservatori. Per quanto riguarda i liberali danesi $(\mathrm{Vd})$, nonostante la loro collocazione sull'area della destra moderata (ma con un punteggio inferiore rispetto ai partiti conservatori e liberal-conservatori), in considerazione della loro storia, base sociale e tradizione politica è sembrato più coerente collocarli nella «famiglia dei partiti agrari» tipica dei sistemi politici del Nord Europa (Thomas 1988; Smith 1988).

Ovviamente ragionare in termini di comportamenti concreti, di collocazioni spaziali, di orientamenti di policy, oltre che di affinità ideologiche, apre anche la questione della distinzione tra partiti conservatori «secolari» e partiti conservatori «confessionali». Non a caso, infatti, nei principali studi condotti sui partiti conservatori, prima ricordati, hanno trovato spazio anche i grandi partiti democristiani continentali. Entrambe le famiglie partitiche sono accomunate dalla funzione di salvaguardia del sistema delle diseguaglianze nella distribuzione del potere e nell'accesso alle risorse (Caciagli 1986).

Tuttavia, qui abbiamo preferito tenere i due gruppi ben distinti. Innanzitutto perché, ad esclusione della Csu bavarese (con un punteggio di 7,8) e della Csv lussemburghese (il cui punteggio è di 7,4$)$, tutti i grandi partiti democristiani continentali hanno una collocazione più moderata (dal 5,4 della Dc al 6,7 della Cdu: tab. 1). In sostanza, sono più partiti di centro che di destra moderata. In secondo luogo, perché l'aggregazione tra destra conservatrice e destra confessionale «è comunque persuasivamente criticata dalle recenti ricerche sociologiche che

10 Proprio con riferimento a questi partiti Smith (1988) usa l'espressione «liberalconservatori». In uno dei lavori più completi e sistematici sui partiti liberali (Kirchner 1988) si propende per considerare questi partiti, comunque, appartenenti alla «famiglia liberale» (su questa posizione si vedano, anche, Castles e Mair 1984 e Lane e Ersson 1987), pur ammettendo un loro spostamento a destra per le politiche economiche, meno marcato peraltro per le issues sociali: per il Vvd si veda Daalder e Koole (1988); per il Prl e il Pvv Rudd (1988); per l'Fpö Luther (1988). Alcuni commentatori, invece, hanno collocato l'Fpö tra i partiti di «estrema destra» (Ignazi 1994) o «neo-populisti» (Taggart 1995; Lane e Ersson 1996). In un recente lavoro di taglio elettorale sui partiti di centro-destra europei negli ultimi cinquant'anni abbiamo preferito classificare tutti questi partiti nella «famiglia liberale» (Raniolo 1998). 
mostrano come i partiti cristiano-democratici influenzano il loro ambiente politico e sociale in modo molto differente dai partiti conservatori» (Kalyvas 1996, 321). In effetti, e siamo ad un terzo punto, i partiti cristiano-democratici (discorso diverso si deve fare per le formazioni politiche protestanti del Nord Europa) sono, insieme ai partiti socialdemocratici, espressione di quella «tradizione social-riformista» tipicamente europea incarnata dai «partiti inclusivi interclassisti» (Schmidt 1992, 174).

Precisata in questo modo la scelta dei casi comparabili (i partiti distinti per affinità ideologiche) e l'ambito della ricerca (i paesi e l'arco di tempo) non ci resta che precisare su quali proprietà (o variabili) dei partiti abbiamo centrato l'attenzione. A tal fine, abbiamo preso in considerazione le «tre principali dimensioni della loro azione politica: l'arena elettorale, l'organizzazione interna e l'impatto sulle istituzioni statali» (Calise 1992, 13). Il ricorso a più dimensioni d'analisi ci consente, da un lato, di superare il semplice «elettoralismo» ${ }^{11}$ e, dall'altro, di puntualizzare che i partiti, come del resto ogni organizzazione complessa, sono fenomeni poliedrici. A queste dimensioni abbiamo dedicato rispettivamente $i$ tre successivi paragrafi.

\section{I partiti conservatori nell'arena elettorale}

Nella figura 1 sono rappresentati i valori aggregati delle percentuali di voto delle principali famiglie ideologiche di centrodestra (abbiamo escluso i partiti agrari dell'Europa settentrionale) articolati in tre periodi (1945-61, 1962-79 e 1980-96).

Della figura 1 è sufficiente sottolineare due aspetti. In primo luogo, le dimensioni elettorali della destra europea sono in gran parte determinate dall'apporto dei partiti confessionali e di quelli conservatori, con un ruolo subordinato dei partiti liberali. In particolare, da un lato risalta la rilevanza elettorale dei partiti democratico-cristiani delle democrazie dell'Europa continentale e delle piccole democrazie del Benelux: la Dc, la Cdu/Csu, l'Övp, la Csv, il Psc/Cvp e la Cda, che complessivamente registrano percentuali medie di consensi che oscillano tra il $34 \%$ e il $45 \%$. Dall'altro, la consistenza di alcuni grandi partiti conser-

11 Per cui i cambiamenti elettorali aggregati sarebbero condizioni necessarie e sufficienti tanto del cambiamento dei partiti che del cambiamento dei sistemi partitici (Mair 1989). 


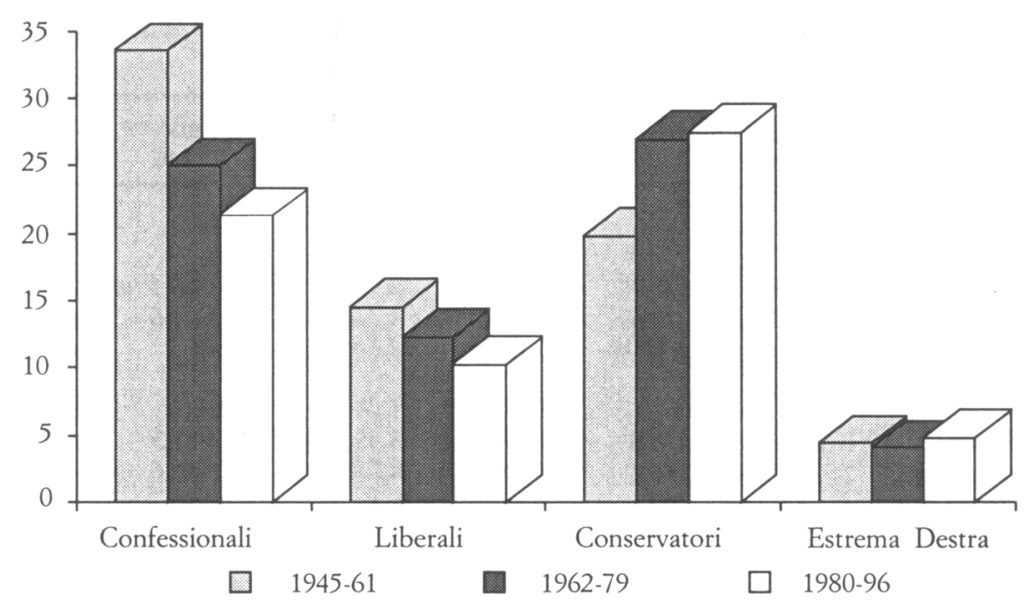

FIG. 1. Dimensioni elettorali delle principali famiglie ideologiche di destra in Europa occidentale (1945-1996) (medie \% di periodo).

vatori: i Conservatori inglesi, il Fianna Fàil in Irlanda e i partiti conservatori delle tre giovani democrazie del sud Europa (il $\mathrm{Pp}$ in Spagna, Nd in Grecia e il Psd in Portogallo) e della Francia: il campo di oscillazione delle percentuali di voto medie di questi partiti varia dal $32 \%$ per il $\mathrm{Pp}$ al $45 \%$ del Fianna Fàil.

In secondo luogo, la figura 1 mette in evidenza l'esistenza di una sostanziale specularità tra le sorti elettorali delle due anime principali (quella confessionale e quella conservatrice) della destra europea. Specularità, che come vedremo meglio più avanti, è il riflesso della sovrapposizione tra una ben determinata distribuzione geografica e una distribuzione ideologica della forza elettorale dei partiti di destra. Al di là del carattere aggregato dei dati, è certo, però, che nel passaggio da una fase all'altra, tutti i partiti confessionali mostrano, chi più chi meno, segni di declino (tab. 2). L'esito di questo deflusso di suffragi è solo parzialmente indicato dalla perdita complessiva (cioè per l'intera famiglia), in meno di quarant'anni, di circa 12 punti percentuali $^{12}$.

$12 \mathrm{La}$ percentuale media di consenso elettorale per i partiti confessionali europei è: $33,6 \%$ per il periodo $1945-1961 ; 25 \%$ per il periodo $1962-79 ; 21,3 \%$ per il periodo 1980-95. Mentre la deviazione standard per i tre periodi è rispettivamente $17,6 \%$, $17,5 \%$ e $15,1 \%$. 
TAB. 2. Forza elettorale delle principali famiglie ideologiche europee per singoli paesi (1945-1996) (medie \% di periodo)

Comunisti Socialisti e Confessio- Agrari Liberali Conserva- Estrema Socialdem. nali tori Destra

$1945-1961$

\begin{tabular}{|c|c|c|c|c|c|c|c|}
\hline $\begin{array}{l}\text { Gran Bretagna } \\
\text { Irlanda } \\
\text { Grecia } \\
\text { Portogallo } \\
\text { Spagna }\end{array}$ & & $\begin{array}{l}46,7 \\
10,6\end{array}$ & 27,4 & & 5,9 & $\begin{array}{l}46,1 \\
44,6\end{array}$ & \\
\hline Francia & 25,6 & 18,8 & 19,1 & & 14,5 & 11,3 & 3,5 \\
\hline Norvegia & 5,8 & 46,2 & 9,3 & 8,7 & 11,1 & 18,6 & \\
\hline Danimarca & 5,3 & 40,2 & & 23,4 & & 16,7 & \\
\hline Finlandia & 22,0 & 25,8 & 0,2 & 23,2 & 5,7 & 14,9 & \\
\hline Svezia & 5,7 & 46,2 & & 12,1 & 19,9 & 15,9 & \\
\hline Austria & 4,7 & 42,6 & 45,1 & & & 9,2 & \\
\hline Belgio & 5,6 & 35,0 & 43,8 & & & 12,2 & \\
\hline Germania & 3,9 & 31,5 & 49,3 & & 10,5 & & 11,9 \\
\hline Italia & 31,7 & 21,5 & 41,5 & & 4,3 & & 7,5 \\
\hline Lussemburgo & 8,6 & 32,2 & 41,4 & & 16,6 & & \\
\hline Olanda & 6,7 & 29,3 & 50,6 & & & 8,8 & \\
\hline $1962-1979$ & & & & & & & \\
\hline Gran Bretagna & & 41,4 & & & 13,1 & 41,5 & \\
\hline Irlanda & & 14,4 & 33,5 & & & 47,7 & \\
\hline Grecia & 10,8 & 19,4 & & & 16,3 & 48,1 & 6,8 \\
\hline Portogallo & 16,6 & 33,4 & 11,8 & & & 30,9 & 0,5 \\
\hline Spagna & 10,0 & 29,9 & & & & 34,8 & 7,1 \\
\hline Francia & 24,3 & 21,3 & & & 20,1 & 32,6 & 0,6 \\
\hline Norvegia & 0,9 & 48,0 & 10,5 & 10,0 & 6,6 & 20,7 & 3,5 \\
\hline Danimarca & 2,2 & 42,4 & 3,5 & 16,8 & & 18,8 & 13,8 \\
\hline Finlandia & 18,9 & 25,7 & 2,1 & 18,8 & 5,2 & 17,3 & 5,1 \\
\hline Svezia & 4,8 & 45,4 & 1,6 & 19,5 & 13,4 & 15,0 & \\
\hline Austria & 1,3 & 47,7 & 44,4 & & & 5,8 & \\
\hline Belgio & 3,4 & 27,1 & 33,5 & & & 17,1 & 1,4 \\
\hline Germania & 0,3 & 42,6 & 46,8 & & 7,9 & & 1,9 \\
\hline Italia & 28,8 & 11,5 & 38,6 & & 4,0 & & 6,5 \\
\hline Lussemburgo & 9,3 & 29,1 & 34,9 & & 18,8 & & \\
\hline Olanda & 5,2 & 27,5 & 38,7 & & & 12,7 & 0,4 \\
\hline $1980-1996$ & & & & & & & \\
\hline Gran Bretagna & & 30,9 & & & 21,9 & 42,2 & \\
\hline Irlanda & & 10,6 & 32,3 & & & 44,0 & \\
\hline Grecia & 10,9 & 43,2 & & & 1,5 & 41,6 & 0,4 \\
\hline Portogallo & 13,3 & 29,8 & 8,0 & & & 39,9 & 0,6 \\
\hline Spagna & 7,6 & 41,7 & & & & 30,3 & \\
\hline
\end{tabular}


ТАВ. 2. (segue)

\begin{tabular}{lrccrrrr}
\hline & Comunisti & $\begin{array}{c}\text { Socialisti e } \\
\text { Socialdem. }\end{array}$ & $\begin{array}{c}\text { Confessio- } \\
\text { nali }\end{array}$ & Agrari & $\begin{array}{c}\text { Liberali } \\
\text { tori }\end{array}$ & $\begin{array}{c}\text { Conserva- Estrema } \\
\text { Destra }\end{array}$ \\
\hline Francia & 12,7 & 24,5 & & & 20,0 & 20,1 & 8,2 \\
Norvegia & 0,2 & 44,4 & 8,8 & 9,1 & 3,4 & 25,3 & 6,8 \\
Danimarca & 0,9 & 43,6 & 2,2 & 14,1 & & 23,2 & 6,4 \\
Finlandia & 11,3 & 25,3 & 2,9 & 20,0 & 0,8 & 20,6 & 5,5 \\
Svezia & 5,5 & 43,3 & 3,7 & 10,6 & 9,7 & 21,5 & 3,9 \\
& & & & & & & \\
Austria & 0,5 & 40,7 & 34,3 & & & 15,3 & \\
Belgio & 1,1 & 26,8 & 26,5 & & & 21,4 & 3,8 \\
Germania & 1,8 & 37,6 & 44,6 & & 8,9 & & 1,1 \\
Italia ${ }^{1}$ & 26,9 & 10,4 & 32,3 & & 2,6 & 20,8 & 15,0 \\
Lussemburgo & 4,4 & 29,0 & 34,5 & & 18,8 & & \\
Olanda & 3,5 & 29,6 & 30,5 & & & 18,4 & 0,6 \\
\hline
\end{tabular}

Note:

1 In Italia, la media del periodo $1980-96$ per la famiglia confessionale fa riferimento esclusivo ai voti ottenuti dalla DC. Sono quindi escluse le elezioni del $1994 \mathrm{e}$ del 1996.

Fonti: Katz e Mair (1994); Beyme (1987). «Quaderni dell'Osservatorio Elettorale», «European Journal of Political Research», «West European Politics», «Keesing's Record of World Events»: numeri vari.

Per contro, se volgiamo la nostra attenzione alle vicende politico-elettorali dei partiti conservatori è facile cogliere l'evoluzione di questo raggruppamento politico: nel quindicennio dell'immediato dopoguerra esso era sulla difensiva (un atteggiamento che rifletteva il radicale discredito della destra diffuso non solo nei paesi che avevano sperimentato una parentesi autoritaria ma anche nelle democrazie rimaste liberali); a partire dalla fine degli anni ' 70 lancia invece una vigorosa offensiva ${ }^{13}$ capace di ribaltare lo stato di minorità nel quale si era trovato fino ad allora. Assenti dai paesi ad egemonia cattolica dell'Europa continentale, i partiti conservatori hanno visto crescere il loro peso medio complessivamente di oltre 7 punti percentuali, passando dal 24\% (nel 1945-61) al 31,5\% (nel 1962-79 e nel 1980-96). Con una dispersione che è andata via via riducendosi (anche se non di molto), i valori della deviazione standard sono

13 Sul punto è illuminante il confronto tra le antologie di Layton-Henry (1982) e di Girvin (1988). 
rispettivamente per i tre periodi: $15 \%, 13 \%$ e $10 \%$. Gli ultimi sedici anni - anni del dilagante consenso neo-liberista e della reazione neo-conservatrice al cedimento degli assetti istituzionali keynesiani - hanno così rappresentato il periodo di maggior successo elettorale per i partiti conservatori.

Vediamo i dati con più attenzione. Nella tabella 2 abbiamo indicato, sempre per $i$ tre periodi, le percentuali medie di voto aggregate per $\mathrm{i}$ principali partiti e raggruppamenti ideologici presenti nei sistemi politici dell'Europa occidentale. Essa evidenzia inoltre sia il peso che i partiti hanno nei singoli paesi (in questo modo consente di correggere la fallacia ecologica), sia i rapporti di forza tra le diverse componenti interne all'area politica di destra (in questo modo affronta, per così dire, la fallacia ideologica). La tabella 2, infine, è stata costruita in modo tale da enfatizzare la contrapposizione tra paesi dove predomina una «destra secolare liberal-conservatrice» o, per contro, una «destra confessionale democristiana».

Gli anni che vanno dal 1945 al 1961 (primo riquadro della tabella 2), fatta eccezione per la Gran Bretagna e l'Irlanda, fecero registrare livelli di consenso piuttosto bassi per i partiti conservatori. Lo scarto percentuale tra le medie di consenso dei conservatori inglesi e irlandesi - $46 \%$ per i primi e $44,6 \%$ per i secondi - fu di oltre 30 punti percentuali rispetto ai valori dei conservatori francesi o scandinavi. Se poi il confronto si estendesse anche ai partiti liberal-conservatori (Fpö, Prl/Pvv e Vvd) il dislivello elettorale crescerebbe di circa altri 10 punti percentuali; unica eccezione, come si vede dalla tabella 2, era costituita dalla Venstre danese con un livello medio di consenso pari al $23,4 \%$.

Accomunati dalle dimensioni elettorali, i due partiti conservatori anglosassoni si differenziarono nettamente per le caratteristiche del loro contesto competitivo. In Inghilterra lo spazio politico sostanzialmente dicotomico fu egemonizzato sulla sinistra da un forte partito laburista che deteneva un livello di sostegno elettorale appena di qualche frazione di punto percentuale in più dei conservatori $(46,7 \%)$. In Irlanda, invece, lo spazio competitivo si presentò centrato sulla questione nazionale e, in pratica, vide il confronto piuttosto duro tra un partito predominante, il Fianna Fàil, e il Fine Gael (27,4\%), mentre sulla sinistra si trovava un isolato, almeno in questa prima fase, Partito laburista $(10,6 \%)$.

Tra gli altri paesi, da un lato, risaltavano due sistemi multi- 
partitici polarizzati, Francia e Finlandia, che mostravano un centro-destra in balia di più formazioni - in Finlandia, il partito agrario (Kesk) con il $23 \%$, i liberali (Lkp) con 5,7\%, i protestanti (Skl) con lo $0,2 \%$ e i conservatori (Kok) con il $15 \%$; in Francia, la «palude» centrista aveva raccolto oltre il 30\% (circa il $19 \%$ per l'Mrp e il $14 \%$ per le altre formazioni centriste) mentre i Gollisti hanno avuto un andamento altalenante, dal $22 \%$ del 1951 al 4,3\% del 1956, per risalire al $17,6 \%$ con le prime elezioni della nuova Repubblica. Dall'altro, le democrazie stabili del Nord Europa erano caratterizzate dalla presenza di un partito predominante sulla sinistra (la famiglia dei partiti socialdemocratici raccoglieva oltre il $46 \%$ dei voti in Norvegia e Svezia e poco più del $40 \%$ in Danimarca), mentre i partiti conservatori si collocavano al secondo posto in Norvegia (con il $18,6 \%$, davanti ai liberali e alle formazioni rurali) e al terzo posto in Svezia (con il 16\%, preceduto dai liberali con il $20 \%$ e seguito dal partito agrario con il $12 \%$ ) e Danimarca (con il $17 \%$, preceduto di oltre 6 punti percentuali dai liberali della Venstre).

D'altra parte, nel quindicennio immediatamente successivo al secondo conflitto mondiale l'estremismo di destra ebbe un certo peso solo in Germania, con una media del 2,3\% che comprendeva un insieme di piccoli partiti nati già durante l'occupazione alleata; in Francia, con una media di periodo del 3,5\%, anche questa relativa alle varie componenti dell'estremismo di destra (il petenismo, il neofascismo e il poujadismo); infine, troviamo l'Italia con il 7,5\% (la media di periodo fa riferimento tanto ai voti dell'Msi che dei Monarchici).

Un'ulteriore conferma del fatto che, in questa prima fase, i partiti conservatori si adattarono con una certa fatica alle trasformazioni politiche indotte dalla guerra mondiale si può ricavare, a contrario, dai successi elettorali dei partiti democristiani dell'Europa continentale che non solo uscirono vittoriosi dal secondo conflitto bellico, ma anche riuscirono con successo ad assorbire l'elettorato moderato (Farneti 1979). In paesi come l'Italia, l'Austria e la Germania federale il «cattolicesimo politico passò da area di difesa della Chiesa ad area di assorbimento dell'opinione popolare e moderata, infine a ruolo egemone che razionalizzò l'area di centro-destra perché capace di congiunzioni» (ibidem, 130; corsivo nostro). Quanto ai dati elettorali, il campo di variazione delle percentuali di consenso per le formazioni confessionali europee fu contenuto in appena 9 punti percentuali: il 41,4\% del Csv in Lussemburgo, e il 50,6\% nel caso 
dell'Olanda (ma questo dato aggrega i valori di due formazioni protestanti, Arp e Chu, e di una più grande cattolica, Kvp) segnano i due estremi nell'ambito dei quali sono ricompresi i valori delle altre formazioni democratico-cristiane europee.

Tra i primi anni ' 60 e la fine degli anni ' 70 il panorama cambia radicalmente (secondo riquadro della tab. 2). I fatti più rilevanti sono stati due, ed entrambi intrecciano dinamica istituzionale e dialettica politica, transizioni di regime e performances dei partiti. Ci si riferisce, per un verso, all'avvento della Quinta Repubblica francese e all'insieme di effetti a cascata che ciò ha comportato per il sistema di relazioni tra i partiti; dall'altro, all'ondata di democratizzazioni che a metà degli anni '70 ha investito l'Europa del Sud. Si è trattato cioè di due sequenze di «crisi-transizione-instaurazione-consolidamento» (Morlino 1998) che, per quanto qualitativamente diverse - nel caso francese la transizione è stata «da democrazia a democrazia» negli altri casi, invece, «da autoritarismo a democrazia»-, hanno avuto una comune conseguenza politica: l'affermazione di un rilevante partito conservatore che, non solo ha occupato con successo lo spazio politico di centro-destra contribuendo così alla stabilizzazione $\mathrm{e}$ alla semplificazione della competizione interpartitica, ma ha anche finito per guidare il processo di consolidamento del nuovo regime (Morlino 1998; Pappas 1996) ${ }^{14}$. In altri termini, potremmo considerare questi partiti come una sorta di «equivalenti funzionali», sul piano politico ed istituzionale, dei partiti democristiani del secondo dopoguerra.

$\mathrm{Ci}$ troviamo di fronte a delle formazioni partitiche di tipo pigliatutto, sostanzialmente pragmatiche e di governo, con ampie basi di consenso moderato e tendenzialmente anticomunista (Morlino 1988). La percentuale di consensi varia dal 32,6\% dei gollisti, al 31\% del Psd portoghese, al 35\% dell'Ucd spagnola (Ap, in questa fase ancora su posizioni estremiste, guadagna il $7,2 \%$ ), per arrivare alla cifra record del $48 \%$ registrata da $\mathrm{Nd}$. Inoltre, questi livelli di consenso non hanno trovato pari tra

14 In breve, sembra possibile estendere a tutti questi casi un'ipotesi che Morlino riferisce esplicitamente alla Grecia e alla Spagna e, solo parzialmente, al Portogallo: «l'instaurazione democratica è più facile, rapida e indolore se è condotta da forze conservatrici di destra che evidentemente svolgono un ruolo di garanti che il processo di cambiamento non supererà certi limiti e soprattutto non porterà anche a rilevanti trasformazioni sociali» (Morlino 1988, 779-780). Altra questione è poi se le forze protagoniste della transizione e dell'instaurazione riuscirono, o meno, a capitalizzare i frutti del loro impegno (ibidem, 780). 
quelli ottenuti dagli altri partiti di centro-destra e di sinistra (con l'eccezione dei socialisti portoghesi che avevano una media di voti del 33,4\%).

Per quanto riguarda gli altri casi, è opportuno distinguere quei sistemi politici dove i partiti conservatori hanno giocato un ruolo centrale da quelli dove, invece, la loro posizione è stata più marginale. In Inghilterra, nel confronto tra primo e secondo periodo, $i$ conservatori hanno perso circa il $4 \%$ e tuttavia hanno mantenuto inalterati i rapporti di forza con i laburisti. Ad avvantaggiarsi di questa situazione di stallo è stato il Partito liberale (con una media del $13 \%$ dei voti, pari a $+7,2$ punti percentuali rispetto al periodo precedente). Tuttavia, in conseguenza dell'efficacia manipolativa del sistema plurality, i liberali non sono riusciti a capitalizzare questo ricavo in termini di seggi parlamentari e di aspettative di accesso al governo. In Irlanda, invece, con un incremento di circa 3 punti percentuali rispetto al periodo precedente il Fianna Fàil è rimasto saldamente il primo partito sia nell'elettorato che al governo - fatta eccezione per il gabinetto di coalizione tra Fg e Laburisti del 1973 (Budge 1995).

Anche nelle democrazie dove la forza elettorale dei partiti conservatori è stata più modesta $i$ trend non sono caratterizzati da univocità (tab. 2). I risultati elettorali dei partiti conservatori del Nord Europa sono stati, in gran parte, il riflesso della crescente turbolenza delle arene elettorali e delle più ampie trasformazioni che tra la fine degli anni '60 e il decennio successivo hanno segnato questi sistemi partitici. Turbolenze e pressioni che, a differenza del caso britannico, sono state fedelmente registrate dall'operatività di sistemi elettorali proporzionali e si sono scaricate principalmente sui tradizionali partiti di governo socialdemocratici o di centro. Mentre hanno visto nell'offerta di destra conservatrice o, addirittura radicale e populista (si pensi al ruolo negli anni '70 dei Partiti del progresso danesi e norvegesi), un'occasione di espressione.

In particolare, i paesi scandinavi hanno registrato una contenuta crescita del consenso per i partiti conservatori, in media attorno ai 2 punti percentuali. Unica eccezione è stata quella dell'Msp svedese che ha subito una lieve flessione - va, anche, segnalato il caso della Danimarca dove la crescita elettorale del Kf (+2 punti) si è accoppiata al crollo della Venstre che ha perso ben 7 punti percentuali. Per le formazioni liberal-conservatrici del continente hanno finito, invece, per prevalere degli anda- 
menti positivi: di circa 4 punti percentuali sono cresciuti l'Fpö austriaco e il Vvd olandese, e di circa 5 punti i belgi del Prl/Pvv.

Né va dimenticato che tra i primi anni ' 60 e il decennio successivo, specie nelle democrazie scandinave, i partiti conservatori hanno dovuto affrontare una pericolosa sfida rappresentata dall'estremismo di destra. In particolare, risalta il caso del Partito del progresso danese (Frpd) che ha ottenuto circa il 13,8\% e quello dell'omologo partito norvegese (Frpn) che, però, ha conseguito appena il $3,5 \%$.

L'ultimo riquadro della tabella 2 ci mostra la configurazione più recente dei sistemi partitici delle sedici democrazie dell'Europa occidentale. Tra i partiti conservatori si registra soltanto un ingresso: il «partito-azienda» di Berlusconi. Nelle elezioni del 1994, approfittando del collasso delle forze politiche centriste e di governo, con il $21 \%$ dei voti validi Forza Italia si afferma sulla scena politica italiana diventando un partito di maggioranza «relativissima» ${ }^{15}$. Anche nel caso italiano, non diversamente da quanto si è detto prima per la Francia, gli aspetti politici si intrecciano con gli aspetti istituzionali ${ }^{16}$ dando luogo, pur nella continuità del genere democratico, ad una «transizione di regime» tuttora in corso e dagli effetti ancora incerti.

In secondo luogo, è evidente la progressiva crescita del peso elettorale e politico dei partiti conservatori scandinavi. Tutti e quattro i partiti si collocano adesso al di sopra della soglia del $20 \%$ con in testa l'Ho norvegese (oltre il $25 \%$ ) e il Kf Danese (circa il $23 \%$ ). Parimenti rilevante è la crescita di popolarità dei partiti (liberal-)conservatori in Belgio, Olanda e Austria ${ }^{17}$.

Quanto agli altri partiti, alcuni mostrano dei cedimenti anche consistenti nei livelli di sostegno elettorale. È il caso dei Gollisti $(-2,9 \%)$, del Fianna Fàil $(-3,8 \%)$ e di Nuova democra-

15 Sia nel senso che lo scarto dal secondo partito, il Pds, è piuttosto risicato (appena lo $0,6 \%)$, ma anche perché alla maggioranza nell'elettorato $(21 \%)$ non corrisponde la maggioranza in parlamento $(15,7 \%)$. Nella graduatoria dei partiti parlamentari $\mathrm{Fi}$ si colloca al quarto posto dopo la Lega, il Pds e An (Morlino 1996).

16 Sia per le radicali trasformazioni del sistema partitico (la competizione tra partiti passa da un modello multipolare a uno bipolare) sia per i mutamenti delle regole del gioco (l'adozione di un sistema elettorale misto a prevalenza maggioritaria).

17 Se dalla media di periodo (1980-1996) passiamo ai risultati delle singole elezioni le performances dell'Fpö austriaca sono ancora più stupefacenti. Essa ha addirittura superato la soglia del $22 \%$ sia nelle elezioni del '94 che in quelle del ' 95 . L'esito di questa crescente popolarità elettorale ha alterato i rapporti di forza tra l'Övp e l'Fpö. In poco più di un decennio lo scarto tra le percentuali di consenso dei due partiti è passato da ben 38 punti percentuali ad appena 5 punti. Il che è, forse, il preludio di un sorpasso a destra. 
zia $(-6,5 \%)$. Gli altri presentano invece una crescita. È questo il caso dei Conservatori inglesi $(+0,7 \%)$, del Partito socialdemocratico portoghese $(+9,1 \%)$ e del Partito popolare spagnolo (che se negli anni '70 si trovava su posizioni estremiste, adesso si colloca nell'area di governo con una percentuale di consensi $\operatorname{del} 30,3 \%$ ).

Infine, lo spostamento a destra che a partire dagli anni ' 80 ha caratterizzato l'opinione pubblica europea ha comportato l'affermazione di diverse formazioni di estrema destra, più neopopuliste che neo-fasciste. Accanto agli altalenanti andamenti elettorali dei Fronti del progresso scandinavi, va ricordato il successo della Nyd svedese che nelle elezioni del 1991 ottenne il $6,7 \%$ - però, già nelle elezioni del '94 avrebbe subito un calo di oltre 5 punti. Più consistenti sono, invece, le percentuali di voto per il Fn di Le Pen che già nel 1993 con oltre il 12\% aveva sorpassato il Pcf. Anche in Italia negli anni '90 il peso dei partiti di estrema destra è cospicuo: con oltre il 13\% troviamo An che però in questi anni si è progressivamente spostata su posizioni più moderate; mentre la Lega nord, che nelle ultime elezioni del 1996 guadagnò oltre il 10\%, sperimenta un processo opposto di radicalizzazione.

Proviamo, adesso, a tirare le somme di quanto detto grazie a due semplici indicatori: le dimensioni elettorali, misurate dai livelli medi di consenso di periodo; e l'evoluzione elettorale, misurata dai saldi netti tra le medie di periodo (1945-61 e 198096). Questi valori sono stati rappresentati in un diagramma (fig. 2). Al fine di facilitare il commento lo abbiamo diviso in quattro quadranti. Ciò ci permette di distinguere (dicotomizzando) $\mathrm{i}$ vari partiti a seconda delle dimensioni elettorali in piccoli, collocati nei due riquadri in basso, o grandi, collocati nei riquadri in alto. E, ancora, a seconda del tipo di evoluzione elettorale in partiti in declino collocati nei riquadri a sinistra, e partiti in crescita collocati in quelli a destra. La griglia analitica così ottenuta consente di tracciare una mappa delle fortune elettorali dei partiti europei di destra.

Innanzitutto, sotto un profilo statico la figura 2 riconferma la netta distinzione tra democrazie dell'Europa continentale, alle quali vanno aggiunte Gran Bretagna e Irlanda, tutte collocate nei riquadri superiori, e le democrazie dell'Europa settentrionale posizionate nella parte inferiore del diagramma. $\mathrm{Ma}$ anche dalla considerazione degli scarti di periodo emergono delle informazioni interessanti. In sostanza, con la rilevante eccezione 


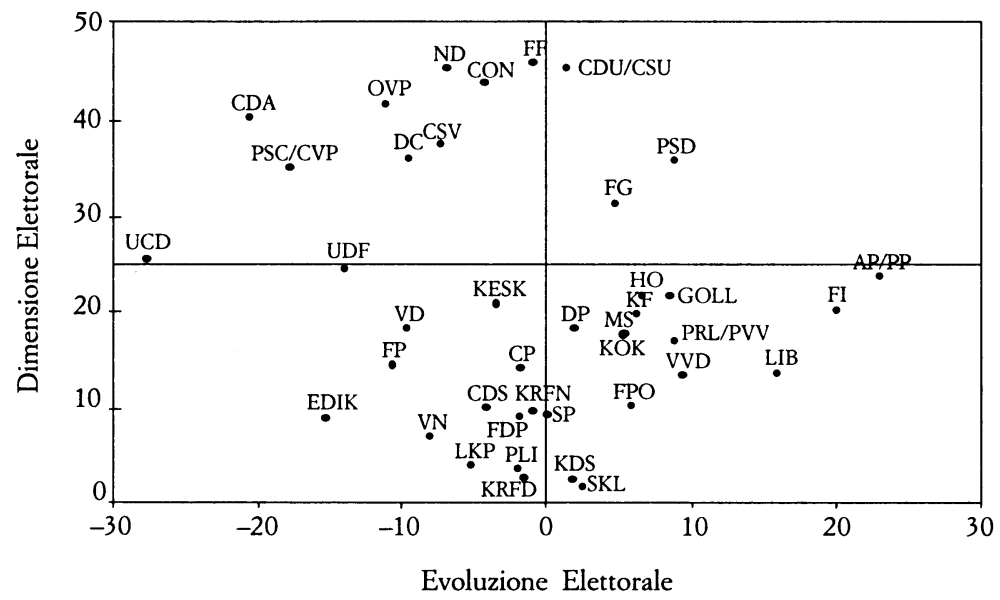

FIG. 2. Dimensioni ed evoluzione elettorale dei partiti di destra europei (1945-1996) (valori percentuali).

della Cdu/Csu tedesca e dell'Fg irlandese, sono proprio i partiti confessionali che, nel corso dell'ultimo mezzo secolo, hanno sperimentato il maggiore declino tra i partiti di destra. Un vero e proprio terremoto elettorale coinvolge la $\mathrm{Cda}$ olandese $(-20$ punti percentuali), le due formazioni belghe del Psc/Cvp (-17), l'Övp austriaca $(-11)$ e la Dc italiana $(-9)^{18}$. Caso a parte costituiscono i partiti protestanti nel Nord Europa, che registrano scarti positivi sia pure piuttosto contenuti ${ }^{19}$.

In secondo luogo, tra i partiti conservatori possiamo distinguere un primo gruppo di tre partiti (i Conservatori britannici, Fianna Fail e Nea Demokratia) che godono di una base di consensi relativamente stabile e, nonostante qualche cedimento elettorale $(-3,9$ punti in percentuale per i Conservatori, $-6,4$ per $\mathrm{Nd}$, e $-0,6$ per $\mathrm{Ff}$ ), dopo cinquant' anni sono elettoralmente ancora i primi della famiglia. Un secondo gruppo è ben individua-

18 Il dato della Dc non tiene conto dei risultati delle elezioni del 1994 e del 1996. Comunque, forzando un po' la logica politica, se si prendono come riferimento i voti ottenuti in quelle due occasioni dal Partito popolare $(\mathrm{Pp})$ lo scarto balza a circa -15 punti percentuali.

19 In particolare, due partiti registrano degli scarti positivi e gli altri due degli scarti negativi - rispettivamente: $+2,7$ punti percentuali per l'Skl finlandese; $+2,1$ punti per il Kds svedese; $-0,5$ punti percentuali il Krf norvegese; $-1,2$ punti il Krf danese. 
to dai quattro partiti scandinavi (Ho, Kok, Msp e Kf) e dalle formazioni liberal-conservatrici continentali (Fpö, Vvd e Prl/ $\mathrm{Pvv})$ che, invece, registrano tutti dei cospicui incrementi delle rispettive basi di consenso ${ }^{20}$. Infine, troviamo il Psd portoghese, il Pp spagnolo e i gollisti francesi, tre partiti le cui vicende elettorali sono state tumultuose (la deviazione standard per le tre formazioni politiche è, infatti, di circa il $10 \%$ per i Gollisti, di quasi l' $8 \%$ per i socialdemocratici portoghesi e addirittura del $12,4 \%$ per il Pp spagnolo) e che, nonostante tutto, nell'ultimo periodo hanno accresciuto le loro percentuali di voti (mediamente di circa 9 punti).

Come si diceva, dunque, questi dati sottolineano l'esistenza di una marcata specularità tra le fortune elettorali delle due principali famiglie ideologiche di centro-destra, quella confessionale e quella liberal-conservatrice. A ben guardare, però, questa specularità può voler dire due cose ben distinte che analiticamente vanno tenute separate. In primo luogo, da una prospettiva «individuale», relativa cioè alle vicende elettorali dei singoli partiti, esiste una marcata relazione negativa tra gli andamenti elettorali dei due raggruppamenti ideologici. In secondo luogo, da un punto di vista «sistemico» e «storico-genetico» (Bartolini 1986), quegli andamenti evidenziano l'esistenza di una sostanziale incompatibilità di famiglia ideologica tra partiti espressione della destra secolare e della destra denominazionale.

In genere, in quei paesi dell'Europa continentale dove la formazione dei partiti confessionali di massa ha avuto successo si è assistito irrimediabilmente alla decadenza del «conservatorismo politico» (Beyme 1987). Si tratta, per lo più, di processi collegati con l'avvento delle «prime democratizzazioni» (Dahl 1980 ) e con il conseguente superamento della «soglia dell'incorporazione» (Rokkan 1982). D'altra parte, solo in tempi recenti si sono verificati dei casi in cui l'occupazione dello spazio politico-elettorale di un partito (neo-)conservatore ha finito per ridurre il ruolo o, addirittura, per eliminare del tutto i partiti confessionali. Sarebbe proprio questo il caso della Quinta Repubblica in Francia e anche delle transizioni democratiche sperimentate negli anni '70 nel Sud Europa.

20 I dati sono rispettivamente $+6,5$ punti percentuali per il $\mathrm{Kf}$ danese (nei nostri dati abbiamo considerato anche $\mathrm{i}$ valori del $\mathrm{Cd}$, una formazione scissionista dal $\mathrm{Kf}$, $+5,7$ per il Kok finlandese, $+6,8$ per l'Ho norvegese e $+5,6$ per l'Msp svedese. 


\section{Iscritti e modelli organizzativi}

Le principali conseguenze organizzative delle trasformazioni alle quali si accennava nei paragrafi precedenti sono state la progressiva «americanizzazione» dei partiti politici europei, e, da un diverso punto di vista, la diffusione di un «contagio da destra» (Epstein 1967, 257 e ss.). L'esito, però, non è stato tanto l'affermazione di una nuova best way organizzativa - per esempio, dal partito di massa al partito professionale-elettorale (Panebianco 1982) - ma, piuttosto, quello di una diversità dei modelli organizzativi. Adesso «nei vari sistemi di partito si continua a riscontrare la presenza di una pluralità dei tipi di partito, ciascuno incline a sfruttare le posizioni acquisite e le conseguenti rendite di posizione» (Pasquino 1997, 127).

Ma come si collocano i partiti conservatori rispetto a questi trend organizzativi? Per rispondere al quesito guarderemo agli andamenti della membership e ai modelli organizzativi. Vediamo, innanzitutto, l'aspetto relativo alle iscrizioni.

Nonostante tutto quello che si è detto e scritto riguardo al «declino organizzativo» dei partiti (Katz e Mair 1994; Ignazi 1996) ci sono ancora svariati motivi per considerare il tema della membership meritevole di attenzione. Alcuni sono motivi di interesse generale, cioè che valgono un po' per tutti i partiti. In effetti, gli iscritti continuano a costituire una risorsa organizzativa cruciale ${ }^{21}$ per procacciarsi la quale i partiti usano, in genere, una combinazione di diversi tipi di incentivi, materiali, di solidarietà e purposive (Clark e Wilson 1961). Anzi, si potrebbe avanzare l'ipotesi che la stessa crescita dei tassi di membership (cioè del rapporto tra iscritti ad un partito ed elettori) dipende, proprio, dalla disponibilità di incentivi di vario genere (intesi come aspettative di benefici futuri) a disposizione del partito $\left(\right.$ Ware 1996) ${ }^{22}$.

21 Permangono, infatti, almeno quattro buone ragioni che spiegano perché i partiti continuino ad impiegare molte delle loro energie e sforzi nel procacciare iscritti e militanti: primo, la base di membership costituisce un bacino sicuro, perché fuori mercato, di voti; secondo, a prescindere dal ruolo del finanziamento pubblico dei partiti, i membri sono rilevanti come fonte complementare di emolumenti; terzo, gli iscritti possono fungere da serbatoio per il reclutamento dei candidati; infine, sono anche una importante risorsa nella competizione intra-partitica (Müller e Steininger 1994, 66-67; Ware 1996, 63-64).

22 Con l'importante specificazione che gli «incentivi collettivi» creano lealtà ed identificazioni più resistenti rispetto all'adesione opportunistica basata sugli «incentivi selettivi». 


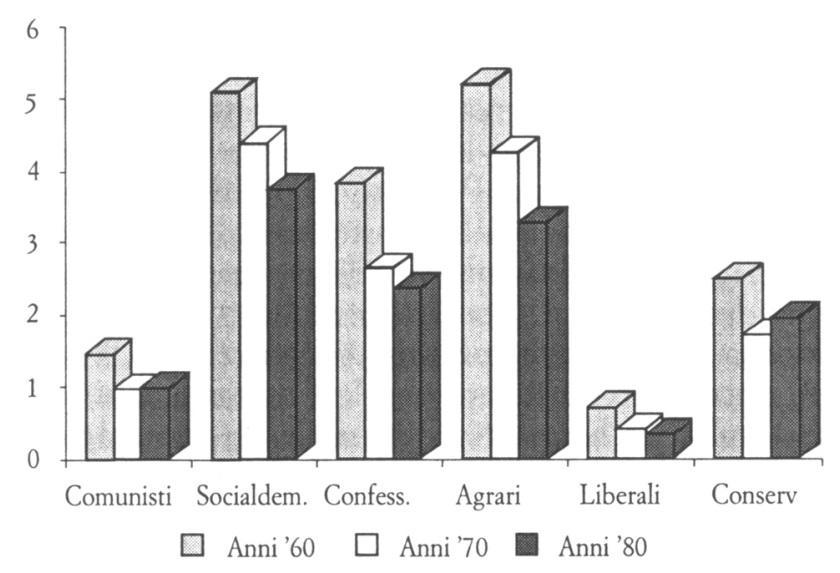

FIG. 3. Tassi di membership (M/E) per le principali famiglie ideologiche europee (1960-1990) (valori percentuali).

Dalla figura 3, dove sono stati rappresentati i valori aggregati per le singole famiglie politiche dei tassi di membershi $p^{23}$, risaltano due rilevanti informazioni. In primo luogo, i partiti conservatori sono gli unici che mostrano una crescita del livello di iscritti in rapporto alle dimensioni degli elettorati nazionali. Tuttavia, più che il dato aggregato sono significativi i singoli casi. Ad esclusione del Partito conservatore britannico (1.500.000 iscritti in meno dagli anni '60 ad oggi; una «presenza sociale» ridottasi di circa 4 punti percentuali) e dei tre partiti dell'Europa settentrionale (il Kf danese, il Kok finlandese e il Msp svedese), tutti gli altri partiti registrano una crescita della rispettiva densità organizzativa. Quelli che presentano il maggiore incremento del tasso di membership ${ }^{24}$ sono i Gollisti fran-

$23 \mathrm{E}$ forse il caso di ricordare che gli studiosi hanno utilizzato due diversi indici per analizzare l'incidenza delle iscrizioni. Il primo, utilizzato originariamente da Duverger (1961), è il rapporto tra iscritti e votanti per il partito (M/V); il secondo, al quale facciamo riferimento in queste pagine, è dato dal rapporto tra iscritti e aventi diritto al voto o elettori $(\mathrm{M} / \mathrm{E})$. I due indici hanno suscitato ampie discussioni. Comunque sia, il primo $(\mathrm{M} / \mathrm{V})$ può essere concettualizzato come un indicatore efficace della capacità di integrazione organizzativa del partito; il secondo $(\mathrm{M} / \mathrm{E})$, invece, indica una più generale capacità di penetrazione sociale del partito (Raniolo 1997, cap. 4).

$24 \mathrm{Ci}$ riferiamo al cosiddetto «tasso di cambiamento della membership», ovvero al rapporto tra lo scarto dei tassi di membership degli anni '80 e '60 $\left(\mathrm{M} / \mathrm{E}_{\mathrm{t}}+\mathrm{n}-\mathrm{M} / \mathrm{E}_{\mathrm{t}}\right)$ e il tasso di membership iniziale $\left(\mathrm{M} / \mathrm{E}_{\mathrm{t}}\right)$. Per un breve commento di questo indice rimandiamo a Katz, Mair et al. (1992, 331-332). 
cesi $(+106 \%)$ e i partiti conservatori delle democrazie del Sud Europa, in ordine di rilevanza Psd $(+427 \%), \mathrm{Pp}(+260 \%)$ e $\mathrm{Nd}$ $(+123 \%)^{25}$. Parimenti in crescita sono i tassi di cambiamento delle membership dei partiti liberal-conservatori delle democrazie continentali (i belgi del Prl/Pvv, l'Fpö austriaco e gli olandesi del $\mathrm{Vvd}$ ) ma non delle altre formazioni liberali che, invece, mostrano inequivocabili segni di declino organizzativo.

Questi dati, come si diceva, potrebbero essere spiegati ricorrendo alla teoria degli incentivi organizzativi (Ware 1996). In breve, potremmo sostenere che le trasformazioni socio-economiche e culturali hanno reso possibile un più ampio ricorso da parte dei partiti conservatori a quella fonte di «sostegno diffuso», nel senso eastoniano, rappresentata dai purposive incentives (Clark e Wilson 1961), cioè degli incentivi connessi agli appelli ideologici e/o ai programmi di policy. D'altra parte, la maggiore espansione della base di aderenti è anche associata a quei partiti (Gollisti, Partito socialdemocratico, Nuova democrazia) che, dalla loro posizione dominante, hanno ottenuto un notevole «plusvalore» politico ed organizzativo: in termini di incumbency, di patronaggio delle cariche, di compenetrazione con le istituzioni. E ciò, dalla prospettiva della teoria degli incentivi, può essere visto come un'espressione della possibilità e/o volontà di questi partiti di ricorrere a degli incentivi selettivi (siano essi materiali o di status) come mezzo per costituire delle cospicue riserve di «sostegno specifico».

In secondo luogo, il confronto dei tassi di membership tra le diverse famiglie ideologiche costituisce una spia, certo semplificata ma ciò nondimeno illuminante, dei tipi di partito, siano essi concepiti come «tipi storici» o «tipi analitici» (Sartori 1965). La questione, a dire il vero, era stata già posta perentoriamente da Duverger $(1961,112)$ : la distinzione tra partiti di quadri e partiti di massa corrisponde, all'incirca, a quella tra destra e sinistra, tra partiti «borghesi» e partiti «proletari» ${ }^{26}$.

25 La base degli iscritti del Psd è cresciuta nell'arco di circa quindici anni di oltre sette volte, passando dalle circa 20.000 unità nel 1975 alle 143.000 degli anni ' 90 . Ben più consistenti sono gli aumenti degli iscritti in valore assoluto per la $\mathrm{Nd}$ greca, che passa da 20.000 unità nel 1977 a 400.000 nel 1991, e per il Pp spagnolo, che nel 1989 conta 262.000 aderenti contro i 164.000 del 1982 e i 60.000 del 1977. Quanto al Partito gollista basti ricordare che secondo alcune stime da 86.000 iscritti nel 1965 (ma erano 7.000 nel 1955) si è passati a 750.000 nel 1985. Forza Italia, l'altro partito neoconservatore preso in esame, oscilla attorno ai 300.000 iscritti ai «Club Azzurri» (stima al 1994, riportata in Morlino 1996).

26 Ovviamente il confronto quantitativo tra tassi di membership non deve far di- 
La figura 3 ci dice, così, che sono i partiti della «famiglia socialista» che presentano la più elevata proporzione di elettori nazionali che sono formalmente iscritti al partito. Il tasso di membership è, infatti, del $4,4 \%$; e, proprio con riferimento a questi partiti Wellhofer (1979) ha parlato di capacità di «incapsulazione organizzativa ${ }^{27}$.

D'altra parte, una significativa presenza sociale è tipica non solo dei partiti agrari scandinavi $(4,3 \%)$, e soprattutto del Partito di centro (Kesk) finlandese - il cui tasso di membership è di circa l' $8 \%$-, ma anche dei partiti confessionali, specie dei grandi partiti democratico-cristiani dell'Europa continentale. Sono gli austriaci dell'Övp che, con un tasso di membership pari all' $11 \%$, si presentano come uno dei partiti europei con maggiore densità organizzativa; segue la Dc italiana con circa il $5 \%$, i belgi del Psc/Cvp (circa 3\%), la Cda olandese (circa 2\%) e, infine, la Cdu tedesca (circa l' $1 \%$ ).

In questa speciale classifica i partiti conservatori sono al terzo posto con il $2 \%$. Non mancano, però, vistose variazioni interne a tale famiglia ideologica. Innanzitutto, i partiti conservatori elettoralmente e governativamente più forti mostrano tutti una cospicua densità organizzativa ${ }^{28}$. Un secondo gruppo di partiti dimensionalmente medi ( $\mathrm{Fi}, \mathrm{Pp}, \mathrm{Psd}$ e $\mathrm{Rpr}$ ) fa registrare valori del rapporto iscritti/elettori più esigui ${ }^{29}$. Infine, i partiti conservatori scandinavi, nonostante siano elettoralmente meno forti, presentano dei tassi di membership piuttosto significativi, probabilmente riflesso dell'elevata «associabilità partigiana» (Morlino 1995) che caratterizza questi sistemi politici ${ }^{30}$.

menticare che tra i diversi tipi di partito cambia profondamente anche «la [stessa] natura della partecipazione» (Duverger 1961, 172-182).

27 Il concetto di «incapsulazione organizzativa», così com'è usato da Wellhofer (1979), con riferimento ai casi dei partiti socialisti argentino, inglese, norvegese e svedese, è definito operativamente da tre indicatori: a) l'articolazione periferica del partito; $b$ ) la proporzione di elettori iscritta al partito; c) la proporzione di elettori che è anche iscritta alle organizzazioni economiche del partito.

28 I tassi di membership sono rispettivamente del $4 \%$ per i Conservatori inglesi, del $3,6 \%$ per $\mathrm{Ff}$ e del $3,4 \%$ per $\mathrm{Nd}$.

29 In questo gruppo Forza Italia, con circa lo $0,6 \%$, si trova a fare da fanalino di coda, preceduta dal Partito popolare spagnolo $(0,7 \%)$ e dal Partito socialdemocratico portoghese $(0,9 \%)$, mentre con l' $1,5 \%$ troviamo i gollisti dell'Rpr. Su questi livelli sono anche i valori del rapporto tra iscritti ed elettorato nazionale per l'Fpö austriaco $(0,6 \%)$ e il Vvd olandese $(0,7 \%)$, mentre il valore per i belgi $\mathrm{Prl} / \mathrm{Pvv}$ è circa il doppio (1,5\%).

30 Tali valori sono del $2,5 \%$ per il $\mathrm{Kf}$ danese, del 2,4\% per il Kok finlandese, dell' $1,4 \%$ per l'Msp svedese, e addirittura del $4,6 \%$ (vero e proprio record per l'intera famiglia politica) per l'Ho norvegese. 
In definitiva, la figura 3 mostra i limiti della bipartizione di Duverger tra «partiti di quadri» e «partiti di massa». Tanto i partiti confessionali che quelli conservatori (per tacere dei partiti rurali) sfuggono alla presa dello schema duvergeriano finendo così per rappresentare degli «ibridi» (Epstein 1967). Da qui l'esigenza di rivedere la stessa tipologia. Nei termini di Sartori, i partiti conservatori rientrerebbero nella classe dei «partiti elettorali di massa», un tipo organizzativo intermedio tra i «partiti intermittenti d'élite» e $\mathrm{i}$ «partiti organizzativi di massa» (1965, 41-47). Da parte sua, Caciagli li definisce, insieme ai grandi partiti confessionali continentali, «partiti di iscritti»: partiti cioè che hanno perso le principali «funzioni» del partito di massa della tradizione europea, ma che hanno conservato e potenziato gli aspetti «strutturali» (1983, 28-31). Tuttavia, a complemento del ragionamento di Caciagli, si può ricordare che le radicali trasformazioni organizzative che hanno sperimentato negli ultimi tre decenni i partiti politici riguardano non solo gli aspetti funzionali ma anche, se non soprattutto, quelli strutturali (Katz e Mair 1994 e 1995; Ignazi 1996; Beyme 1996).

Comunque sia, nonostante l'importanza che la membership riveste nelle organizzazioni a carattere associativo, la nozione di modello organizzativo non si esaurisce in essa. Per contro, quando parliamo di «tipo di partito» occorre fare riferimento ad altre dimensioni organizzative (Ersson e Lane 1987), tanto interne (struttura, grado di centralizzazione e coesione) che esterne (relative ai rapporti con la società civile e con le istituzioni). Ed è proprio a questo insieme di caratteristiche infra- e inter-organizzative che si riferiscono etichette quali «partito elettorale-professionale» (Panebianco 1982), «moderno partito di quadri» (Koole 1994), «partito minimo» (Beyme 1996), «partito-cartello» (Katz e Mair 1995), «partito post-moderno» (Beyme 1996), per ricordare solo le più note. In questo quadro i «tassi di adesione», per usare l'espressione di Duverger (1961), ben al di là del loro significato letterale forniscono degli indizi preziosi sulla natura e le trasformazioni dei tipi di partito. Per esempio, in prima approssimazione, consentono di distinguere tra partito di elettori (con un rapporto iscritti/elettori basso) e partito di iscritti (con un rapporto più alto).

D'altra parte, quando passiamo a considerare le caratteristiche strutturali e strategiche che caratterizzano i partiti conservatori, al di là del riconoscimento di una generica «flessibilità organizzativa» - che Peele (1988) considera un aspetto tanto na- 
turale quanto paradossale (per un partito, appunto, «conservatore») del Partito conservatore britannico - che li accomuna, è opportuno distinguere tra i partiti conservatori storici, i conservatori inglesi, irlandesi, le formazioni politiche delle democrazie scandinave (Ho, Kf, Kok, Msp) e i partiti liberal-conservatori (Fpö, Vvd, Prl/Pvv) da un lato e i partiti neo-conservatori (Rpr, Ucd, Ap/Pp, Psd, Nd e Fi) dall'altro.

Le principali caratteristiche del modello organizzativo dei partiti conservatori «storici» sembrano riflettere le caratteristiche del loro imprinting organizzativo. Innanzitutto, non diversamente da quanto accade per gli altri partiti di origine parlamentare come i liberali, i partiti conservatori hanno una discreta autonomia dei parlamentari rispetto al partito «macchina». Il caso in cui quest'aspetto risalta con più forza è proprio il Partito conservatore inglese, «un partito dominato dal gruppo parlamentare a sua volta subordinato al leader e al suo entourage» (Panebianco 1982, 242). La stessa struttura del partito basata su tre pilastri - le «associazioni di base» (constituency associations) coordinate a livello nazionale dalla National Union, il vero e proprio apparato amministrativo rappresentato dall'Ufficio centrale e, infine, il «partito parlamentare» che ha il compito di eleggere il leader - tenuti assieme, specie dopo la svolta thatcheriana, dal ruolo del leader, riflette questo «cleavage» organizzativo.

In secondo luogo, e questa volta a differenza dei partiti liberali, i conservatori si sono caratterizzati per l'esistenza di un cospicuo «grado di organizzazione», nel senso di Janda $(1980)^{31}$. A tal proposito, i partiti conservatori scandinavi sono, sia per l'ampiezza della base di aderenti (iscritti) sia per l'articolazione dell'organizzazione periferica, emblematici: alla fine degli anni '80 si contavano circa 1.000 sezioni per l'Ho norvegese, 850 per l'Msp svedese e 1.200 per il Kok finlandese. E, addirittura, non è mancato chi ha sostenuto che, in questi paesi, «non c'è nessun motivo per distinguere tra partiti di massa socialisti e partiti di quadri non-socialisti» (Sundeberg 1994, 163). L'esistenza di una forte organizzazione, oltre che di una consistente membership, caratterizza, infine, anche l'Fpö austriaco, il Vvd olandese

31 In particolare, delle due facce della densità organizzativa, la prima relativa «all'estensione» periferica e della membership, la seconda «all'intensione» nel senso del grado di coinvolgimento e della rigidità dei legami interni (Janda 1980, 98 e ss.), i partiti liberal-conservatori sembrano più propensi ad attenuare la seconda (si veda supra). 
e il Prl/Pvv belga. Anzi, proprio queste caratteristiche organizzative differenziano i partiti «liberal-conservatori» da quelli «liberal-radicali» (Smith 1988).

In terzo luogo, lo sviluppo organizzativo dei partiti conservatori, spesso avvenuto attraverso una «penetrazione territoriale»(Eliassen e Svaasand 1975), nonostante sia stato accompagnato dalla gravitazione nell'orbita del governo ha finito per produrre una «forte istituzionalizzazione interna» (Panebianco 1982, 106-107). Questa sovrapposizione tra sviluppo organizzativo e ruolo dominante al governo è stato caratteristico dei Conservatori inglesi. Ma ha anche caratterizzato il Fianna Fàil. Così, non solo il partito di De Valera ha rappresentato il naturale partito di governo della Repubblica irlandese ma, a differenza dei Laburisti e del Fine Gael, ha dato vita ad una capillare macchina organizzativa (nel 1985 si contavano 2.500 sezioni locali, le cosiddette cumann) che costituisce tuttora un formidabile collettore di consensi.

Veniamo adesso al modello organizzativo dei partiti «neoconservatori» evidenziando alcuni aspetti idealtipici che ci serviranno da criteri di comparazione dei singoli casi empirici: il Partito socialdemocratico portoghese, l'Unione del centro democratico spagnola, la Nuova democrazia in Grecia, i gollisti e, più di recente, Forza Italia. Innanzi tutto, l'imprinting organizzativo di tutti e cinque questi partiti mette in risalto lo stretto nesso tra sviluppo organizzativo e consolidamento democratico. $\mathrm{Al}$ riguardo, sia pure con riferimento al solo caso della Nuova democrazia greca, Pappas $(1994,4)$ ha parlato della «logica organizzativa come esigenza istituzionale». In altri termini, il successo dell'istituzionalizzazione del partito costituisce una condizione necessaria (per quanto non sufficiente) per far fronte alle due principali sfide che devono affrontare i regimi in transizione: la ricostruzione o riforma delle istituzioni democratiche e la creazione della «legittimità diffusa», come riserva originaria di sostegno al regime (Morlino 1998).

Per quanto riguarda quest'aspetto due casi meritano un veloce richiamo sia pure per motivi diversi. Il primo, un vero e proprio «rompicapo» politologico, è dato dall'Unione del centro democratico spagnola che, nonostante il ruolo politico-istituzionale strategico ricoperto, nel giro di pochi anni crollò - il partito di Suárez passò dal 35\% del 1979 al 7\% del 1982 e si sarebbe sciolto qualche mese dopo quella data - anche come riflesso della scarsa coesione organizzativa interna (Caciagli 1986; 
Colomer 1995). Il secondo caso, quello di Forza Italia di Berlusconi, nonostante il continuo richiamo al modello del «partito leggero» o al «partito del leader», ha messo in evidenza l'importanza della strutturazione organizzativa e del conseguente dilemma democrazia-efficienza - specie se il partito si trova all'opposizione.

In secondo luogo, questi partiti hanno conquistato subito una posizione dominante (a livello elettorale, parlamentare e di governo) nei rispettivi sistemi politici tale da favorire una loro forte compenetrazione con gli apparati statali (Pappas 1996). In altri termini, ci troviamo di fronte a dei casi emblematici di partiti che «sperimentano il processo di istituzionalizzazione da una posizione centrale anziché periferica, partiti che conquistarono il governo nazionale dopo la nascita (e prima che fosse avvenuto il consolidamento organizzativo) e che rimasero al governo per periodi di tempo [più o meno] lunghi» (Panebianco 1982, 211).

Anche per quanto riguarda questo secondo aspetto occorre rilevare alcune eccezioni. Fi, per esempio, è rimasta al governo (con il Polo delle libertà) appena sette mesi e ciò non ha certo favorito l'istituzionalizzazione del partito. Per contro, il Psd portoghese è andato al governo per la prima volta nel 1980, dopo una serie di governi presidenziali, e vi sarebbe rimasto in coalizioni - ora di centro-destra (con il Cds), ora di sinistra (con il Psp) - o da solo fino al 1995, allorquando è stato sostituito da un governo minoritario socialista. L'Alleanza popolare spagnola di Fraga Iribarne (che nel 1989 ha preso il nome di Partito popolare) è invece arrivata al governo solo nel 1996 dopo che era riuscita ad imporsi come partito della destra moderata conservatrice, ricoprendo il ruolo che era stato dell'Ucd.

In terzo luogo, i partiti neo-conservatori si sono affermati in un contesto di sostanziale crisi e delegittimazione del partito di massa, ossia di una organizzazione forte, territorialmente radicata, fornita di una propria burocrazia interna e, soprattutto, di un'ampia base di iscritti. Un tratto, questo, particolarmente spiccato nei due «partiti antipartito», l'Unr di De Gaulle e Forza Italia di Berlusconi, ma presente anche nell'Ucd spagnola e nella $\mathrm{Nd}$ greca. In un certo senso si potrebbe estendere a tutti i casi le conclusioni alle quali arriva Morlino per la Spagna: «in questo paese troviamo, infatti, partiti caratterizzati dal ruolo preminente dei leader, da un numero limitato di iscritti e da una ridotta organizzazione. Ideologia e polarizzazione sono so- 
stituite dall'importanza cruciale dei media e da un'accentuata personalizzazione della vita politica» (Morlino 1992, 260) ${ }^{32}$.

Infine, tutti e cinque questi partiti sono delle organizzazioni top-down, geneticamente e saldamente legate ad una leadership carismatica: Karamanlis per la Nd, Sà Carniero per il Psd, Fraga Iribarne per l'Ap/Pp, Suarez per l'Ucd, De Gaulle per l'Unr e Berlusconi per Fi. Per riprendere delle categorie concettuali utilizzate di recente (Harmel e Svasand 1993, 73-76), potremmo dire che ci troviamo di fronte a dei leader che, in un modo o nell'altro, riescono a combinare i tratti del «creatore» (dell'organizzazione), del «comunicatore» (dell'ideologia, dell'identità) e della personalità «carismatica», tutti indispensabili nella fase iniziale di vita dei nuovi partiti. In realtà, la personalizzazione della leadership è un fenomeno che investe un po' tutti i partiti indipendentemente dalle affinità ideologiche. Nel nostro caso, riguarda tanto i partiti conservatori tradizionali (si pensi per tutti al ruolo della Thatcher per i Conservatori inglesi o, in tutt'altro contesto, di De Valera per il Fianna Fàil), quanto i partiti liberal-conservatori (emblematico è il ruolo che ha avuto Haider nella svolta populista dell'Fpö austriaco). Ma è nel gruppo dei «nuovi» partiti conservatori che la questione dell'oggettivazione del carisma si fa cruciale per la stessa sopravvivenza del partito.

\section{Partecipazione al governo e salienza istituzionale}

Concesso che i partiti «sono prima di tutto delle organizzazioni, e che pertanto l'analisi organizzativa deve precedere ogni altra prospettiva» (Panebianco 1982, 10; corsivo nostro), va ricordato che «né la conquista di iscritti, né quella di elettori sono fini a sé stanti per i partiti. La loro mira principale è arrivare al potere nello stato» (Beyme 1987, 266) attraverso la competizione per il voto popolare. Ciò è senz'altro vero, ma a certe condizioni, «la principale delle quali consiste nella durata della loro permanenza al governo» (Calise 1992, 32) ${ }^{33}$. A questa se ne

32 E appena il caso di ricordare che la combinazione tra ricorso alle tecnologie mediali e personalizzazione raggiunge il parossismo proprio in Forza Italia aprendo delicati $\mathrm{e}$, in parte, trascurati problemi di teoria della democrazia. Come, del resto, si vede dalle espressioni utilizzate nei primi tentativi d'analisi: «partito-azienda», «partito mediatico», «partito neo-patrimoniale», «partito personale di massa», per ricordare le più note.

33 «Paradossalmente - almeno rispetto ad alcune concezioni oggi in voga - una 
TAB. 3. Permanenza al governo delle principali famiglie ideologiche europee (1945-1996) (durata espressa in mesi)

\begin{tabular}{|c|c|c|c|c|c|c|}
\hline & Comunisti & $\begin{array}{c}\text { Socialisti e } \\
\text { Socialdemoc. }\end{array}$ & Confessionali & Agrari & Liberali & Conservatori \\
\hline Gran Bretagna & & 202 & & & & 351 \\
\hline Irlanda & & 204 & 202 & & & 393 \\
\hline Grecia & & 120 & & & & 131 \\
\hline Portogallo & & 75 & 45 & & & 185 \\
\hline Spagna & & 156 & & & & 721 \\
\hline Francia & 36 & 114 & & & 124 & 326 \\
\hline Norvegia & & 438 & 122 & 122 & 75 & 130 \\
\hline Danimarca & & 347 & 21 & 259 & & 199 \\
\hline Finlandia & 122 & 393 & 48 & 478 & 179 & 138 \\
\hline Svezia & & 413 & 38 & 153 & 108 & 81 \\
\hline Austria & 46 & 511 & 362 & & & 42 \\
\hline Belgio & 22 & 366 & 520 & & & 263 \\
\hline Germania & & 185 & 362 & & 445 & \\
\hline Italia & $21^{2}$ & 227 & $545^{2}$ & & 233 & 7 \\
\hline Lussemburgo & 15 & 279 & 529 & & 368 & \\
\hline Olanda & & 300 & 583 & & & 301 \\
\hline Tot. di periodo & o 45 & 271 & 308 & 253 & 219 & 187 \\
\hline
\end{tabular}

Note:

1 Il dato dei conservatori per la Spagna aggrega i mesi di permanenza al governo dell'Ucd (65 mesi) e del Pp (7 mesi al dicembre 1996).

2 I dati dei comunisti e dei democristiani italiani fanno riferimento al 1992.

Fonti: nostra elaborazione su dati tratti da Keman e Budge (1993); «Keesing's Record of World Events» e «European Journal of Political Research» numeri vari.

possono aggiungere altre due: la prima è relativa al «tipo di governo», definito dal numero dei partiti che compongono l'esecutivo (monopartitici o di coalizione) e dallo status parlamentare dei gabinetti (minoritari, maggioritari, o sovramisura); la seconda attiene al loro «orientamento ideologico» lungo l'asse sinistra-destra (Budge e Keman 1990).

Nella tabella 3 sono stati indicati i valori (espressi in mesi)

democrazia funzionante è il risultato della stabilità dei partiti più che della loro alternanza. (...) L'alternanza resta un requisito della democrazia, ma a patto che non si verifichi con una frequenza tale da vanificare gli sforzi di un partito - o di una coalizione di partiti - per incidere sull'amministrazione statale. L'alternativa dei programmi sembra ridursi a poco se manca il tempo per metterli in pratica» (Calise 1992, 40). 
della permanenza al governo dei partiti appartenenti alle diverse famiglie ideologiche (1945-96). I dati aggregati per le singole famiglie politiche mostrano che i conservatori si trovano al penultimo posto, con una presenza media di circa 16 anni (187 mesi). In questa speciale graduatoria sono preceduti dai liberali con circa 18 anni (219 mesi) e dai partiti agrari scandinavi che hanno una permanenza al governo di 253 mesi (circa 21 anni) e, sulla sinistra, dai partiti della famiglia socialista con una incumbency media di circa 22,5 anni. Nell'ambito della destra, però, risalta soprattutto il dato dei partiti confessionali, la cui «occupazione» del governo è mediamente di circa 26 anni (308 mesi).

Una più attenta lettura della tabella ci induce, però, a differenziare il discorso. I paesi scandinavi rientrano in un primo caso: in media la partecipazione dei conservatori al governo è stata di circa 11 anni (il dato si riferisce ad una permanenza discontinua in governi di coalizione) contro i circa 33 anni dei partiti socialdemocratici, i 21 anni dei partiti rurali e i 10 anni dei liberali - ben più modesta è, invece, la partecipazione al governo dei partiti protestanti (in media circa 5 anni).

La Gran Bretagna, l'Irlanda, la Francia e le democrazie del Sud Europa rientrano, invece, in un secondo gruppo. A partire dal dopoguerra, il Partito conservatore inglese, il Fianna Fàil e il Partito gollista sono stati al governo rispettivamente per 29, 33 e 27 anni (queste cifre, espresse in mesi nella tabella 3, sovrastano sensibilmente quelle di qualunque altro partito $)^{34}$. Nel caso dell'Europa del Sud, da un lato, occorre distinguere le vicende del Psd portoghese e della $\mathrm{Nd}$ greca che sono rimasti al potere rispettivamente per circa 15 e 10 anni. Dall'altro, quelle della Spagna dove, dopo il crollo del 1982 dell'Unione del centro democratico (Ucd), ha giocato un ruolo dominante il Psoe. La durata della permanenza dei socialisti al governo è stata di 156 mesi consecutivi, circa 13 anni, e solo nel 1996 il Partito popolare gli è subentrato al governo.

Abbiamo lasciato per ultimi i casi dei tre partiti liberal-conservatori continentali. L'incidenza temporale della partecipazione al governo per questi partiti è cospicua almeno per il $\operatorname{Pr} /$

34 La rilevanza di questi dati si fa più significativa se consideriamo i periodi di permanenza continuativa (almeno due anni) al governo: per i Conservatori inglesi 13 (1951-64) e 18 anni (1979-1997); per il Fianna Fàil 12 (1957-69), 2 (1977-79) e 6 anni (1987-93); per i gollisti 20 (1958-78) e 2 (1993-95). 
Pvv e il Vvd, meno per l'Fpö: i liberali belgi sono rimasti complessivamente al governo poco meno di 22 anni e quelli olandesi ben 25, mentre i liberali austriaci appena 3 anni e mezzo. Questi semplici dati numerici richiedono, però, un commento più articolato.

Innanzitutto, questi partiti presentano una sostanziale sproporzione tra consenso ottenuto e potere di fatto gestito, non diversamente da quanto accade per la famiglia dei liberali - si pensi per esempio al caso dell'Fdp tedesco, con 37 anni di partecipazione al governo, o al $\mathrm{Dp}$ lussemburghese, con poco meno di 31. In questo quadro un caso anomalo è costituito dall'Fpö austriaco che, fino al 1966, è stato penalizzato dalla politica consociativa tra Övp e Spö e, poi, fino al 1983 dalla prassi dei gabinetti monopartitici (in prevalenza socialisti). Da allora l'Fpö ha partecipato a due governi di coalizione a guida Spö, per complessivi 42 mesi, essendone estromesso dopo la sua svolta radicale e protestataria nel $1986^{35}$.

Fin qui il peso «potestativo» dei nostri partiti è stato valutato in termini di semplice permanenza al governo. $\mathrm{Ma}$, come si diceva, ai fini di una valutazione più realistica è opportuno prendere in considerazione almeno altri due indicatori: la composizione del governo, cioè i rapporti tra governo e parlamento e la natura monopartitica o coalizionale dei gabinetti; e l'orientamento ideologico, cioè l'eterogeneità politica dei gabinetti in termini sinistra-destra.

Quanto al primo indicatore, i partiti conservatori hanno fatto parte di governi monopartitici, in 36 casi (su un totale di 122 governi ai quali hanno partecipato tra il 1945 e il 1996), 25 dei quali maggioritari e 11 minoritari. Negli altri 86 casi hanno partecipato a gabinetti di coalizione: in 31 casi in compagini di governo liberal-conservatrici sovramisura; in 16 casi minoritarie, cioè sprovviste di maggioranza in parlamento; nei 36 casi rimanenti si è trattato infine di coalizioni minime vincenti.

Più significative, però, sono le regolarità che vengono fuori dai dati disaggregati. Innanzitutto, meritano attenzione i dati relativi ai gabinetti monopartitici (sia maggioritari che minoritari) che si concentrano, con poca sorpresa, in quattro democrazie: la Gran Bretagna (con 12 gabinetti), l'Irlanda (con 12), la Grecia

35 Va poi ricordato il caso italiano post-1994, dove la coalizione di centro-destra del Polo delle libertà, guidata da Forza Italia, è stata al governo appena 7 mesi (giugnodicembre 1994). 
(con 5), la Spagna (con 4 gabinetti minoritari guidati dalla Ucd e uno dal Pp) e il Portogallo (con 3). I governi monopartitici, com'è noto, sono caratteristici delle cosiddette «democrazie maggioritarie» (Lijphart 1988) e, in effetti, i nostri casi empirici fanno riferimento al paese che rappresenta la culla del «modello Westminster» di democrazia, e al principale emulatore, per tutta una serie di ragioni storico-culturali ed istituzionali di quel modello. Le tre democrazie del Sud Europa, invece, costituiscono tre casi di efficace consolidamento democratico che, grazie alla sinergia tra arrangiamenti istituzionali (sistemi elettorali e forma di governo) e fattori politici (l'affermazione di partiti «dominanti») (Morlino 1998), hanno imboccato la strada della competizione bipolare.

Vediamo adesso la distribuzione delle altre «formule di governo». Con l'eccezione della Finlandia dove, in generale, prevalgono le coalizioni di governo sovradimensionate, in Svezia, Norvegia e Danimarca ci troviamo di fronte a dei gabinetti monopartitici controllati dai partiti socialdemocratici. In queste democrazie, infatti, il peso dei governi di destra o centro-destra appare piuttosto limitato. In mezzo secolo, sono appena 3 i governi ai quali partecipano i partiti conservatori in Svezia, 7 in Norvegia e Finlandia e 9 in Danimarca. Comunque, si tratta per lo più di «coalizioni minoritarie» (in 12 casi) o di «coalizioni minime vincenti» (in 9 casi); solo in Finlandia si contano 4 coalizioni sovramisura alle quali hanno partecipato i conservatori. Del tutto eccezionale è il caso dell'Hoire norvegese che nel 1981, approfittando della debolezza dei socialdemocratici (Dna), ha formato un governo monopartitico minoritario durato 19 mesi.

I liberal-conservatori belgi e olandesi, come riflesso «strutturale» del carattere consociativo delle «piccole» democrazie continentali, hanno spesso fatto parte di governi di coalizioni. Il Pvv/Prl, complessivamente, ha partecipato a 13 coalizioni minime vincenti e a due coalizioni minoritarie, mentre il Vvd olandese ha fatto parte di 6 coalizioni minime vincenti - i due partiti hanno anche partecipato a tre coalizioni sovramisura, così pure i due governi ai quali ha partecipato l'Fpö austriaco erano di coalizione (con i socialisti). Un dato che però differenzia questo gruppo di partiti dalle altre formazioni conservatrici (e li assimila ai partiti della famiglia liberale) è il loro ruolo sostanzialmente secondario, di partner minori all'interno dei governi.

Quanto, infine, al partito gollista, su un totale di 20 governi esso ha dato vita a sole 4 coalizioni minime e a ben 15 gabinetti 
sovramisura. Questi dati sono semplicemente il riflesso sul piano dei rapporti tra esecutivo e parlamento di una competizione di fatto bipolare - la cosiddetta «quadriglia bipolare» - che vede il polo di destra caratterizzato dalla stretta connessione tra Rpr (gollisti) e Udf (centristi-giscardiani).

Se guardiamo, infine, all'altro indicatore relativo alle «affinità elettive» possiamo distinguere la famiglia dei partiti conservatori in due gruppi. Il primo, quello più ampio, mostra una marcata idiosincrasia ideologica in quanto raccoglie tutti quei partiti che partecipano esclusivamente a gabinetti di destra o centrodestra. E ciò, per inciso, differenzia nettamente i conservatori dalle formazioni democristiane continentali, dai partiti di centro (rurali) dei paesi scandinavi e dai partiti liberali che, invece, hanno una spiccata propensione ad alleanze che tagliano trasversalmente lo spazio politico. In sostanza, per i partiti conservatori la discriminante anti-socialista e anti-comunista oltre ad essere costitutiva dell'identità collettiva si esprime anche in conseguenti comportamenti coalizionali e competitivi. In questo primo gruppo ritroviamo - fatta eccezione per le soluzioni contingenti collegate ad eventi bellici, crisi economiche, fasi di transizione - tutti i partiti della famiglia conservatrice ad esclusione del Vvd, del Prl/Pvv e dell'Fpö ${ }^{36}$. Proprio tali formazioni liberal-conservatrici costituiscono il secondo gruppo, caratterizzato invece dalla partecipazione a «governi consensuali», allargati cioè alle forze politiche di sinistra. In particolare, sono $2 \mathrm{i}$ governi consensuali ai quali partecipa il Vvd (su un totale di 9), e 8 quelli dei quali fa parte il $\mathrm{Prl} / \mathrm{Pvv}$ (su un totale di 18), così come entrambi di coalizione sono $\mathrm{i}$ due governi che hanno coinvolto l'Fpö. Questa versatilità coalizionale tipica dei partiti liberali continentali - ma non dei liberali scandinavi, ad eccezione dell'Lkp finlandese, tutti orientati verso coalizioni di centro-destra o di destra - differenzia nettamente questi partiti dagli altri appartenenti alla famiglia conservatrice aprendo qualche dubbio sulla loro stessa classificazione.

Proviamo, infine, a tracciare un quadro sintetico delle performances dei partiti di destra europei sulla base di due indicatori: la dimensione parlamentare (espressa in seggi) e la parteci-

36 Tra le eccezioni, si deve ricordare la recente coalizione (1993) tra Fianna Fàil e Partito laburista durata 23 mesi e guidata da Reynolds che indicava la crisi del tradizionale «sistema a partito dominante». Ma si possono citare anche il governo portoghese del 1985 (Psp + Psd), guidato da Soares e durato 29 mesi, e il governo greco del 1989 $(\mathrm{Nd}+$ Pasok $+\mathrm{Syn})$, guidato da Zolotas, che è durato appena due mesi. 
pazione al governo (durata in mesi) ${ }^{37}$. Questi due indicatori, che presentano delle forti assonanze con quelli normalmente utilizzati nella definizione operativa del concetto di «partito dominante», ci consentono di valutare in concreto la salienza che $i$ partiti conservatori hanno nei diversi sistemi partitici (Fig. 4). In sostanza, possiamo immaginare l'area ricoperta dal diagramma di dispersione come scomposta in quattro riquadri a seconda della diversa associazione tra le due variabili dicotomizzate (debole/forte dimensione parlamentare e debole/forte permanenza al governo $)^{38}$.

Nel riquadro in basso a sinistra troviamo tutti e quattro i partiti conservatori delle democrazie scandinave (Ho, Kok, $\mathrm{Kf}$ e Msp) per i quali potremmo parlare di partiti conservatori di opposizione. Non è casuale se in loro compagnia troviamo sia $\mathrm{i}$ partiti cristiano-democratici (protestanti) del Nord Europa, sia i partiti liberali più piccoli. Per quanto a partire dai primi anni '80, specularmente alla perdita di egemonia dei partiti socialdemocratici, la loro rilevanza sia cresciuta (in termini elettorali, di iscritti e di partecipazione al governo), nel medio-lungo periodo questi partiti costituiscono ancora l'anello debole della famiglia conservatrice.

I partiti liberal-conservatori delle democrazie consociative presentano parecchie analogie con questo primo gruppo di partiti, almeno per quanto attiene all'ampiezza delle basi parlamentari, ma non per la permanenza al governo. In particolare, è il riferimento a questa seconda dimensione che caratterizza il Vvd e il Prl/Pvv. E proprio questi partiti (insieme al Vd danese e all'Fdp tedesco) hanno dimostrato una notevole capacità di convertire la loro collocazione strategica tra sinistra e destra in plusvalore politico. Da qui la marcata sproporzione tra consenso ottenuto e partecipazione al governo che li caratterizza (riquadro in basso a destra della figura 4).

Nel nel riquadro in alto a sinistra troviamo i partiti neoconservatori dell'Europa del Sud che sono stati, e sono ancora oggi, «essenzialmente al centro del processo elettorale, della gestione del potere politico e della elaborazione e definizione delle stesse

37 Il ricorso alla forza parlamentare piuttosto che alla semplice forza elettorale ci permette di scontare l'effetto manipolativo dei sistemi elettorali.

38 L'interpretazione del diagramma della figura 4 ha concettualmente dei punti in comune con la tipologia dei partiti dominanti proposta da Morlino (1998, fig. 6.3). Si noti, però, che per tracciare la nostra «mappa» abbiamo tenuto conto, per entrambe le dimensioni, anche dei valori più esigui. 


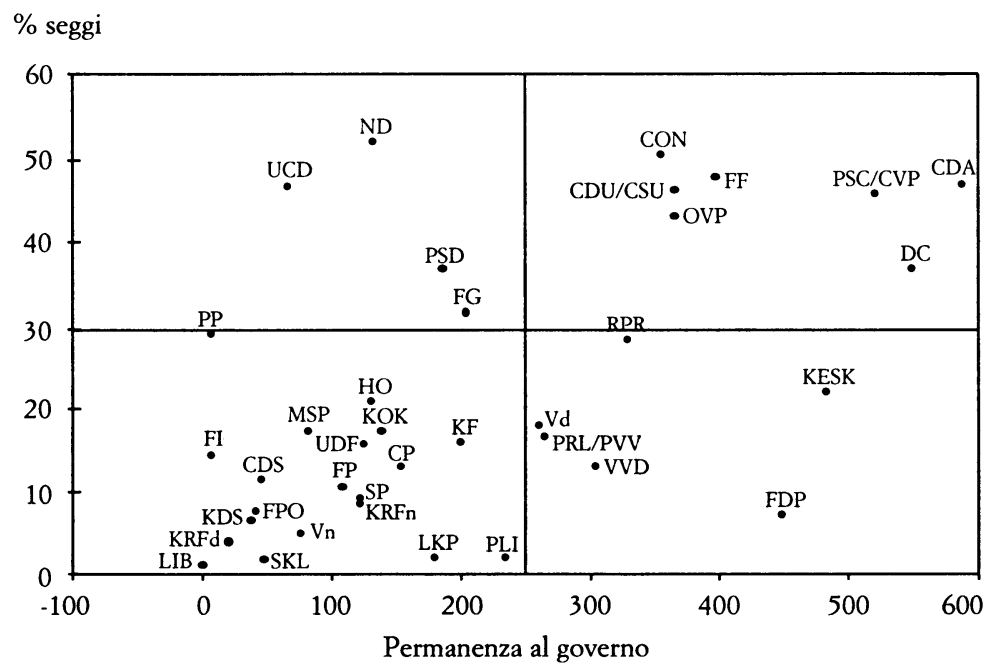

FIG. 4. Relazione tra seggi e permanenza al governo (in mesi) per i partiti di destra europei (1945-1996) (medie \% di periodo).

politiche pubbliche» (Morlino 1992, 243). D'altra parte, il fatto che si affermino sulla scena politica solo a partire della seconda metà degli anni '70 spiega come mai la permanenza al governo di $\mathrm{Nd}, \mathrm{Pp}$, e Psd sia stata più bassa se confrontata con quella degli altri partiti conservatori - $\mathrm{Fi}$, invece, con una percentuale di seggi di circa il $15 \%$ e una durata al governo di appena 7 mesi si trova collocata nel riquadro in basso a sinistra.

Infine, resta il riquadro in alto a destra dove sono collocati $i$ partiti più forti sia per seggi che per durata della partecipazione al governo. Troviamo, infatti, i grandi partiti cristiano-democratici dell'Europa Continentale insieme a quelle formazioni conservatrici che, per usare un'espressione di Peele $(1988,13)$, rappresentano dei naturali partiti di governo: i Conservatori britannici, il Fianna Fàil e, con un certo distacco per taglia parlamentare, il Partito gollista (Rpr). Tre partiti tra loro piuttosto eterogenei e, tuttavia, accomunati dal fatto di competere in un contesto bipolare sorretto o da arrangiamenti elettorali ed istituzionali maggioritari (in tutti e tre i paesi ma, in particolare nella Francia della V Repubblica), o da tradizioni storiche (Kirchheimer parlava dalla «bellezza originaria» della democrazia inglese), o dalla salienza di particolari cleavage (la questione dell'indipendenza nazionale per l'Irlanda). 
Per uno schema di lettura delle performances dei partiti conservatori

Ma come spiegare le performances dei partiti conservatori? Se è vero che nello spazio politico di destra delle democrazie dell'Europa occidentale è in corso un progressivo spostamento (intra-blocco) a favore del raggruppamento ideologico dei partiti conservatori rispetto a quello dei partiti confessionali, come spiegare proprio la prevalenza dei primi?

Per rispondere a questi interrogativi è necessario far ricorso a delle ipotesi interpretative che, sia pure in modo impressionistico, siano in grado di rendere intelligibili le regolarità emerse. Ad un primo livello d'analisi le performances dei partiti politici possono essere considerate come il riflesso di una molteplicità di fattori causali. Per comodità possiamo provare a raggruppare questi fattori in tre famiglie di variabili (Pedersen 1983; Ersson e Lane 1987) collegate tra di loro da relazioni causali ad «imbuto», cioè che vanno dalle cause più generiche e di sfondo a quelle più specifiche e prossime. Comunque, la regola è che passando dai fattori di influenza più remoti e indiretti a quelli più adiacenti e diretti ci si muove verso «condizioni» che rientrano nella sfera di azione e di manipolabilità dei partiti (Sartori 1982). Vediamo quali sono queste variabili:

1) Ad un primo è più generale livello le performances di un partito sono da collegare alle trasformazioni dell'ambiente generale di riferimento. Dalla prospettiva dell'adattamento dei singoli partiti, questi cambiamenti costituiscono delle sfide alle quali i partiti dovrebbero rispondere allo scopo di restare competitivi (Müller e Steininger 1994, 5). I cambiamenti nella struttura occupazionale, nei comportamenti religiosi, nella cultura politica, nei mass media, nonché le pressioni provenienti dall'ambiente internazionale sono tutti fattori che hanno un impatto indiretto sui partiti, sulla loro azione, sulla loro competitività.

2) Ad un secondo livello troviamo gli input e le trasformazioni più prossime e dirette attinenti a quella che seguendo Schlesinger $(1985,1154$ e ss.) possiamo indicare come la «struttura delle opportunità politiche», che definisce quali sono le cariche pubbliche rilevanti per i partiti, le regole per accedervi e i modelli di comportamento idonei alla loro «cattura». In concreto, ci si riferisce al ruolo di variabile interveniente che hanno gli assetti istituzionali - tra le altre, i sistemi elettorali, le forme di governo e di stato, le norme per il finan- 
ziamento dei partiti ma, anche, altre «regole del gioco» relative, per esempio, all'organizzazione degli interessi economici nella formazione degli obiettivi, delle strategie dei partiti e finanche nell'influenzare gli esiti della competizione politica (Steinmo, Thelen e Longstreth 1992).

3) Infine, la terza famiglia di fattori che influenza le performances dei partiti politici rinvia alla capacità di azione strategica del partito nell'ambito di una specifica «struttura della competizione partitica». A tal proposito è stato ricordato che, «il grado di complessità/semplicità [dell'arena elettorale] è collegato a molti fattori ma il fattore principale è l'esistenza o meno di competitori del partito, cioè di altri partiti che peschino nello stesso «territorio di caccia» o che avanzino pretese sulle risorse elettorali fondamentali del partito» (Panebianco 1982, 393). Per contro, qualora due partiti si trovino in una collocazione «di opposizione senza competizione la porzione della base elettorale che rappresenta il domain da cui dipende l'identità del partito non può essere catturata o scalfita dal partito avversario» (ibidem, 193-194).

Alla luce di questo schema, è possibile sostenere, per esempio, che le performances dei partiti conservatori, negli ultimi quindici-vent'anni, siano il riflesso di tutta una serie di trasformazioni favorevoli del loro contesto di riferimento (socio-culturale, economico, internazionale) alle quali essi si sono adattati efficacemente. Chiamiamo questo primo tentativo di spiegazione l'ipotesi dell'ambiente generale di riferimento.

Un esempio eccellente dell'applicazione di questa ipotesi si può ricavare da quegli studi che prendono come punto di partenza le ambivalenze dell'attuale processo di modernizzazione, le sue contraddizioni intrinseche, gli effetti perversi collegati alla transizione dalla società industriale a quella post-industriale (Giddens 1997): l'accelerazione dell'urbanizzazione, lo sviluppo del terziario e della società dei servizi, la dissoluzione dei modelli di identità delle classi lavoratrici, l'impatto delle nuove tecnologie e dei mass media, le radicali trasformazioni nelle relazioni tra i due sessi e tra le generazioni, la secolarizzazione dei comportamenti e l'individualismo, la globalizzazione, e l'elenco non è certo esaustivo ${ }^{39}$.

39 Una versione più recente dell'ipotesi della modernizzazione è quella che potremmo chiamare dell'economia politica della globalizzazione. In breve, «il cambiamento del contesto di political economy è diventato ancora più rapido a partire dalla prima 
Un'altra variante di quest'ipotesi rinvia ai cambiamenti della struttura e/o della salienza dei cleavages. All'originaria configurazione dei cleavages, a partire dal peso della frattura di classe, delle democrazie occidentali si sono sovrapposte altre e nuove linee di divisione quali valori materialisti/post-materialisti, establishment/anti-establishment, modernismo-postmodernismo e, per finire, quella tra gruppi sociali che sono «dentro» (garantiti) e quelli che sono «fuori» (marginali) (Gibbins 1989; Inglehart 1993; Esping-Andersen 1997). Le trasformazioni sono di tale intensità che non solo il conflitto socio-politico ha finito per assumere aspetti inusitati, ma la stessa articolazione binaria (sinistra/ destra) dello spazio politico sembra aver perso di rilevanza (Kitschelt e Hellemans 1990). Il punto da fermare qui è che «vi è stato un pregiudizio teso ad enfatizzare gli elementi progressisti dei mutamenti di valore sottostimando, invece, gli aspetti conservatori-autoritari» (Ignazi e Ysmal 1992, 320). Era legittimo chiedersi, infatti, se quei cambiamenti non «riflettessero, nell'opinione pubblica occidentale, un revival di lungo periodo del conservatorismo piuttosto che una reazione temporanea a una tendenza di lungo-periodo verso sinistra» (Minkenberg e Inglehart 1989, 81).

Tuttavia, l'adesione a questa prima ipotesi, se intesa in modo meccanico, corre il rischio di essere fuorviante oltre che eccessivamente semplificatrice. In effetti, per quanto l'ambiente generale di riferimento possa costituire un terreno fertile sul quale si muovono i partiti conservatori, questo da solo non basta per rendere conto delle loro performances. Tutt'al più costituisce una precondizione favorevole. Detto altrimenti, «i comportamenti politici non sono del tutto vincolati dal contesto, né - certo - possono prescinderne oltre un certo punto» (Pappalardo 1992, 149). Tra le spiegazioni di tipo «strutturale» e quelle centrate sul «volontarismo» c'è spazio per un ampio ventaglio di spiegazioni per così dire «strategiche», cioè che tengano conto tanto delle possibilità di scelta degli attori che dei vincoli.

metà degli anni ' 80 , soprattutto in ragione dei fenomeni prodotti dalla contestuale diffusione delle nuove tecnologie informatiche, dalla crescita del commercio internazionale e dalla internazionalizzazione dei mercati finanziari» (Vassallo 1997, 12). Ora, «al doppio impatto di una più aperta competizione commerciale ed ai rischi di instabilità prodotti dalla globalizzazione dei mercati finanziari» (ibidem, 15-16) i paesi dell'Unione europea hanno risposto con l'istituzionalizzazione della «dottrina della stabilità» che costituisce un elemento cardine del nuovo consenso liberal-conservatore. 
Nell'ambito di questo quadro interpretativo più realistico, le ragioni del successo dei partiti conservatori andrebbero più correttamente ricercate anche, se non soprattutto, a livello dell'ideologia, delle strategie competitive e degli assetti organizzativi. Cioè di quell'insieme di tratti normativi ed organizzativi che hanno consentito ai partiti conservatori di affrontare in modo «soddisfacente» (se non proprio «ottimizzante») le sfide che provengono dal loro ambiente. Chiamiamo questa seconda interpretazione l'ipotesi del vantaggio competitivo.

Come si diceva, questo vantaggio competitivo ha, innanzitutto, un carattere «normativo», ideologico. Il neo-conservatorismo comprende, al proprio interno, elementi diversi e per certi aspetti anche contraddittori: un conto sono gli aspetti liberali dell'individualismo opposto alla massificazione e all'invadenza del pubblico, un conto sono le nostalgie per le società ben ordinate, il recupero dei valori morali tradizionali e l'insofferenza per le diversità culturali e razziali (Ignazi 1994, 246). A tal proposito, già Girvin (1988) distingueva tra «neo-conservatorismo» e «conservatorismo tradizionale» ${ }^{40}$.

Ebbene, proprio, quest'ambivalenza ideologica costituirebbe uno dei principali punti di forza competitivi per i partiti conservatori. In effetti, con la progressiva estensione delle politiche neo-liberiste (a livello economico) e neo-conservatrici (a livello sociale), i partiti conservatori hanno finito per assorbire le basi sociali di riferimento dei partiti liberali - tra l'altro, sempre in bilico tra destra e sinistra via via che dalle issues economiche si passa a quelle civili e sociali (Smith 1988). Inoltre, rispetto ai partiti cristiano-democratici, si sono trovati più attrezzati per affrontare le nuove sfide emergenti. E ciò sia perché, grazie alla prevalenza della variante neoliberista del conservatorismo, meglio di quelli sono riusciti a bilanciare esigenze di valore ed esigenze pragmatiche di governo, sia perché sono stati appena

40 Il primo è popolare e democratico nel senso che cerca di utilizzare i valori di massa e dà loro una rilevanza elettorale. Tende ad essere meritocratico piuttosto che élitista. Il mercato e l'individuo giocano un ruolo centrale nel conservatorismo contemporaneo, mentre una visione più organicista dell'azione era tipica delle altre forme di conservatorismo. [Il neo-conservatorismo] ha, anche, tentato di essere più attivo e radicale nella promozione dei suoi obiettivi. C'è qui una contraddizione tra il tradizionale appello alla stabilità e alla sicurezza e l'attuale promozione del cambiamento con la sua conseguente instabilità. Questa contraddizione è stata bene espressa nella tensione tra conservatorismo morale o dei valori e conservatorismo di mercato e individualistico (Girvin 1988, 10-11; corsivo nostro). 
sfiorati dai processi di secolarizzazione dell'elettorato europeo che, per contro, tanto pesantemente hanno inciso sulle fortune politico-elettorali dei partiti confessionali.

D'altra parte, la «visione del mondo» neo-conservatrice ha finito per riflettersi sostanzialmente anche sul bagaglio ideologico dei partiti socialisti e socialdemocratici. «Ora tutti i partiti credono in un'economia di mercato con qualche correttivo sociale. I partiti socialdemocratici hanno finalmente rigettato le loro rivendicazioni dirette a realizzare un'alternativa al capitalismo» (Paloheimo 1987, 48). Anzi, i partiti di sinistra al potere «hanno di fatto imitato la politica dei conservatori in molti aspetti, dalla sollecitazione dell'iniziativa privata al clientelismo di stato. $\mathrm{Ma}$ ciò che più conta è che è entrata in crisi l'idea stessa di una possibilità di cambiamento radicale, in nome dei principi egualitari, che era stata propria della sinistra. Chi propugna mutamenti prudenti nel quadro della sostanziale conservazione sembra quindi destinato a prevalere» (Caciagli 1993, 63).

Per quanto le «predisposizioni culturali» (Pappalardo 1992, 162) e l'affermazione di un nuovo policy paradigm (Hall 1992) costituiscano due variabili cruciali, le performances dei nostri partiti non si possono ridurre ad esse. Altri fattori vanno presi in esame. Innanzitutto, si può ricordare che i partiti «cercano di esaltare, anche organizzativamente, le loro differenze rispetto ai concorrenti e di sfruttare quelli che ritengono i loro punti forti per raggiungere, mantenere ed eventualmente espandere il loro elettorato» (Pasquino 1997, 127). Se ciò è vero, il fatto che $i$ partiti conservatori presentino dei modelli organizzativi congruenti con le attuali tendenze (strutturali/funzionali) alla «americanizzazione», alla «professionalizzazione», al «contagio da destra», alla «statalizzazione», per ricordare solo le più note, costituisce un ulteriore vantaggio competitivo rispetto ai partiti della tradizionale politica di massa socialista e democristiana (si veda il quarto paragrafo).

Non va dimenticato inoltre il carattere sempre più «estensivo» che hanno finito per assumere le strategie competitive dei partiti nelle democrazie europee. Come ricorda Mair, sulla scia di Kirchheimer, «i partiti abbandonano la loro classe gardée per competere nella società a tutto campo, e rinunciano ad una efficacia in profondità in cambio di una audience più larga e di un successo elettorale più immediato» $(1992,115)$. E evidente come queste strategie competitive siano piuttosto congeniali per dei partiti che si definiscono «interclassisti» $o$, più correttamen- 
te, «aclassisti», partiti «di tutto il popolo» o della «gente» (Girvin 1988; Caciagli 1993; Taggart 1995).

D'altra parte, la corsa al «centro», il richiamo ad una pluralità di forze sociali e ad una generica opinione pubblica moderata hanno degli esiti diversi per i nostri partiti a seconda del contesto competitivo. Cioè, per rifarci allo schema proposto all'inizio di questo paragrafo, a seconda dei loro rapporti con i partiti «competitori» di destra, da un lato, e con i partiti «oppositori» di sinistra, dall'altro (Panebianco 1982).

I dati relativi agli andamenti elettorali e alla partecipazione al governo emersi nei paragrafi precedenti consentono di avanzare la seguente ipotesi: laddove la configurazione dello spazio politico di destra è più «ricca» - cioè, sono presenti partiti appartenenti a tutte le principali famiglie indicate nella tabella 1 più squilibrati sono $i$ rapporti con $i$ partiti di sinistra $e$, complessivamente, più deboli sono le possibilità di successo per $\mathrm{i}$ conservatori. Sembrerebbe essere proprio questo il caso dei partiti conservatori scandinavi ma, anche, dei partiti liberal-conservatori olandesi, belgi e austriaci. Per contro, in tutti gli altri casi - dove la destra è egemonizzata proprio da un partito conservatore che, grazie anche al ruolo di istituzioni di tipo maggioritario ${ }^{41}$, compete secondo uno schema bipolare con un partito socialista o laburista più o meno rilevante - , i rapporti con $i$ principali partiti di sinistra sono più bilanciati, la posta in gioco della competizione è più netta e consistente $\mathrm{e}$, infine, la salienza dei partiti conservatori è complessivamente maggiore.

Ma proprio in questo scenario competitivo i partiti conservatori devono affrontare un nuovo dilemma: perseguire una strategia di massimizzazione dei voti non solo mette in discussione gli impegni ideologici, ma rende ancora più drammatico ed urgente il problema della rappresentanza dei gruppi esclusi o minacciati dai cambiamenti sociali ed economici in atto (le cui domande non coincidono di certo con quelle del cosiddetto «elettore mediano»). Ecco dunque spiegato, a partire dai primi anni '80, lo spostamento di ampi segmenti dell'opinione pubblica moderata, non solo europea, in direzione «neo-autoritaria»

$41 \mathrm{Si}$ potrebbe sostenere, ma il punto meriterebbe un maggiore approfondimento, che le soluzioni istituzionali maggioritarie siano più svantaggiose per le formazioni cristiano-democratiche e, in genere, centriste. Infatti, questi arrangiamenti, dando origine a dalle spinte competitive bipolari, penalizzano tutti quei partiti posizionati al centro dello spazio politico elettorale. 
(Morlino e Mattei 1992), «neo-populista» (Taggart 1995) o, ancora, «autoritaria e tradizionalista» (Ignazi 1994). Nel momento della massima diffusione del consenso neo-conservatore, è paradossale che i partiti conservatori - specie se di governo - siano costretti a confrontarsi con la sfida della protesta e con la conseguente crescita della componente estremista e radicale della destra europea.

\section{Appendice*}

Ap/Pp: Alianza popular [dal 1989 Partido popular] (Alleanza popolare/Partito popolare)

Arp: Anti-revolutionnaire party (Partito anti-rivoluzionario [partito protestante])

Cd: Centrumdemocraten (Centro democratico)

Cda: Christen democratische appel (Appello cristiano democratico [nasce nel 1980 come fusione tra Kvp, Arp e Chu])

Cds: Partido do centro democràtico social (Partito del centro democratico sociale [dal $1995 \mathrm{Pp}$ : Partito popolare])

Cdu/Csu: Christlich demokratische union Deutschlands/Christlich soziale union in Bayern (Unione cristiano democratica tedesca/Unione cristiano sociale bavarese)

Chu: Christelijk historische unite (Unione storica cristiana [partito protestante])

Con: Conservative party (Partito conservatore)

Cp'86: Centrumpartj'86 (Partito di centro '86)

$\mathrm{Cp}$ : Centerpartiet (Partito di centro [partito rurale] svedese)

Csv: Christlich-soziale volkspartei (Partito cristiano sociale)

Dc: Democrazia cristiana [dal 1994 si è divisa in Pp (Partito popolare), Ccd (Centro democratico cristiano) e Cdu (Cristiano democratici uniti)]

Dp: Demokratische partei (Partito democratico [liberale])

Dvu: Deutsche volksunion (Unione del popolo della Germania)

Edek: Enose demokratiku kentrou (Unione democratica di centro)

Epen: Ethniki politiki enosis (Unione politica nazionale)

Fdf: Front démocratique del francophones bruxellois (Fronte democratico francofono)

* Il siglario fa esclusivo riferimento ai partiti europei di destra indicati nella tab. 1. 
Fdp: Freie demokratische partei (Partito democratico della libertà)

Ff: Fianna Fäil (Soldati del destino [partito repubblicano; conservatore])

Fg: Fine Gael (Stirpe dei gaeli [confessionale])

Fi: Forza Italia

Fn: Front national (Fronte nazionale)

Fp: Folkpartiet (Partito liberale)

Fpö: Freibeitliche partei österreichsches (Partito austriaco della libertà)

Frpd: Fremskridtspartiet (Partito del progresso danese)

Frpn: Fremskrittspartiet (Partito del progresso norvegese)

Ho: Hoire (Destra [partito conservatore])

Kds: Kristdemokratiska sambällspartiet (Partito della comunità democratico cristiana)

Kesk: Keskustapuolue (Partito di centro [prima del 1965 Unione agraria])

Kf: Det konservative folkeparti (Partito popolare conservatore)

Krfd: Kristeligt folkeparti (Partito popolare cristiano danese)

Krfn: Kristeligt folkeparti (Partito popolare cristiano norvegese)

Kok: Kansallinen kokoomus (Coalizione nazionale)

Kvp: Katholieke volkspartij (Partito popolare cattolico)

Lib: Liberal party (Partito liberale)

Lkp: Liberaalinen kansanpuolue (Partito popolare liberale)

Msi/An: Movimento sociale (ora Alleanza nazionale)

Msp: Moderata samlingspartiet (Partito moderato)

Nd: Nea demokratia (Nuova democrazia)

Nf: National front (Fronte nazionale)

Npd: Nationaldemokratische partei Deutschlands (Partito nazionale democratico della Germania)

Nyd: Nya demokratie (Nuova democrazia)

Övp: Osterreichsche Volkspartei (Partito Popolare Austriaco)

Pdc: Partido da democracia cristão (Partito cristiano democratico)

Pli: Partito liberale italiano

Prl/Pvv: Parti réformateur libéral/Partij voor vrijheid en vooruitgang (Partito liberale riformatore [vallone]/Partito della libertà e del progresso [fiammingo])

Psc/Cvp: Parti social chrétien/Cristelijke volkspartij (Partito sociale cristiano [vallone]/Partito popolare cristiano [fiammingo])

Psd: Partido social democrata (Partito socialdemocratico [conservatore]) 
Rep: Die Republikaner (Repubblicani)

Rpr: Rassemblement pour la Répubblique (Alleanza per la Repubblica [gollisti])

Sf: Sinn Féin

Skl: Suomen kansan demokraatiinen liitto (Lega democratica popolare finlandese)

Smp: Suomen maaseudun puolue (Partito rurale finlandese)

Sp: Senterpartiet (Partito di centro [partito rurale])

Ucd: Union de centro democratico (Unione del centro democratico)

Udf: Union pour la démocratie française (Unione per la democrazia francese [giscardiani])

Vd: Venstre ([Sinistra] Partito liberale danese)

Vlb: Vlaams blok (Blocco fiammingo)

Vn: Venstre ([Sinistra] Partito liberale norvegese)

Vvd: Volkspartj voor vrijheid democratie (Partito popolare per la libertà e il progresso)

\section{Riferimenti bibliografici}

Barnes, H.S. (1966), Ideology and the Organization of Conflict: On the Relationship between Political Thought and Behavior, in «Journal of Politics», vol. 28, n. 3, pp. 513-530.

Bartolini, S. (1986), Partiti e sistemi di partito, in G. Pasquino (a cura di), Manuale di scienza della politica, Bologna, Il Mulino, pp. 231280.

Bartolini, S. e P. Mair (1990), Identity, Competition, and Electoral Availability: The Stabilisation of European Electorates, 1885-1985, Cambridge, Cambridge University Press.

Bogdanor, V. (1983), Liberal Party Politics, Oxford, Oxford University Press.

Beyme, von K. (1987), I partiti nelle democrazie occidentali, Bologna, Zanichelli.

- (1996), Party Leadership and Change in Party System: Towards a Postmodern Party State?, in «Government and Opposition», n. 2, pp. 135-159.

Budge, I. (1995), Gran Bretagna e Irlanda: variazioni di regime di partito dominante, in J.M. Colomer (a cura di), La politica in Europa, Roma-Bari, Laterza, pp. 33-114.

Budge, I. e H. Keman (1990), Parties and Democracy. Coalition Formation and Government Functioning in Twenty States, Oxford, Oxford University Press.

Caciagli, M. (1983), Declino o persistenza del partito di iscritti, in «Democrazia e Diritto», n. 3, pp. 27-48. 
- (1986), Elezioni e partiti politici nella Spagna postfranchista, Padova, Liviana.

- (1993), L'evoluzione dei partiti conservatori, testo non pubblicato.

Caciagli, M. et al. (1992), Christian Democracy in Europe, Barcellona, Institut de Ciències Politiques i Socials.

Calise, M. (a cura di) (1992), Come cambiano i partiti, Bologna, Il Mulino.

Castles, F.G. e P. Mair (1984), Left-right Political Scales: Some «Expert» Judgments, in «European Journal of Political Research», vol. 14 , pp. 73-88.

Clark, P.B. e J.Q. Wilson (1961), Incentive Systems: A Theory of Organizations, in «Administrative Scienze Quarterly», vol. 6, pp. 129-166.

Colomer, J.M. (1995), Spagna e Portogallo: regimi di leadership di partito, in J.M. Colomer (a cura di), La politica in Europa, RomaBari, Laterza, pp. 283-350.

Daalder, H. e R. Koole (1988), Liberal Parties in the Netherlands, Kirchner (1988), pp. 151-177.

Dahl, R.A. (1980), Poliarchia. Partecipazione e opposizione nei sistemi politici, Milano, F. Angeli.

Dogan, M. e D. Pelassy (1990), How to Compare Nations: Strategies in Comparative Politics, Chatham, Chatham House.

Duverger, M. (1961), I partiti politici, Milano, Edizioni di Comunità.

Eliassen, K.A. e L. Svaasand (1975), The Formation of Mass Political Organizations: An Analytical Framework, in «Scandinavian Political Studies», vol. 10, pp. 95-121.

Epstein, L.D. (1967), Political Parties in Western Democracies, London, Pall Mall Press.

Ersson, S.O. e J.E. Lane (1987), Politics and Society in Western Europe, London, Sage.

Esping-Andersen, G. (1997), La politica senza classe?, in N. Negri e L. Sciolla (a cura di), Il paese dei paradossi. Le basi sociali della politica in Italia, Roma, Nis, pp. 19-51.

Farneti, P. (1979), Partiti, stato e mercato: appunti per un'analisi comparata, in L. Graziano e S. Tarrow (a cura di), La crisi italiana, Torino, Einaudi, pp. 113-175.

Gibbins, J.R. (a cura di) (1989), Contemporary Political Culture, London, Sage.

Giddens, A. (1997), Oltre la destra e la sinistra, Bologna, Il Mulino.

Girvin, B. (a cura di) (1988), The Transformation of Contemporary Conservatism, London, Sage.

Hall, P.A. (1992), The Movement from Keynesianism to Monetarism: Institutional Analysis and British Economic Policy in the 1970s, in Steinmo, Thelen e Longstreth (1992), pp. 90-113.

Harmel, R. e L. Svasand (1993), Party Leadership and Party Institutionalisation: Three Phases of Development, in «West European Politics», n. 2, pp. 67-87. 
Humphreys, P. e M. Steed (1988), Identifying Liberal Parties, in Kirchner (1988), pp. 396-435.

Ignazi, P. (1994), L'estrema destra in Europa, Bologna, Il Mulino.

- (1996), The Crisis of Parties and the Rise of New Political Parties, in «Party Politics», II, n. 4, pp. 549-566.

Ignazi, P. e C. Ysmal (a cura di) (1992), Extreme Right-wing Parties in Europe, in «European Journal of Political Research», vol. 22, n. 1, numero speciale.

Inglehart, R. (1993), Valori e cultura politica nella società industriale avanzata, Padova, Liviana.

Irving, R.E.M. (1979), The Christian Democratic Parties of Western Europe, London, Allen \& Unwin.

Jacobs, F. (1989), Western European Political Parties, London, Longman.

Janda, K. (1980), Political Parties: A Cross-national Survey, New York, The Free Press.

Kalyvas, S.N. (1996), La formazione dei partiti confessionali in Europa, in «Rivista Italiana di Scienza Politica», vol. 26, n. 2, pp. 317-363.

Katz R.S. e P. Mair (a cura di) (1994), How Parties Organize, London, Sage.

Katz, R.S. e P. Mair (1995), Changing Models of Party Organization and Party Democracy: The Emergence of the Cartel Party, in «Party Politics», vol. 1, n. 1, pp. 5-28.

Katz, R.S., P. Mair et al. (1992), The Membership of Political Parties in European Democracies 1960-1990, in «European Journal of Political Research», vol. 22, n. 2, pp. 329-345.

Keman, H. (1995), I «Paesi Bassi»: confronto e fusione in società frammentate, in J.M. Colomer (a cura di), La politica in Europa, RomaBari, Laterza, pp. 351-420.

Kirchner, E.J. (a cura di) (1988), Liberal Parties in Western Europe, Cambridge, Cambridge University Press.

Kitschelt, H. e S. Hellemans (1990), The Left-right Semantics and the New Politics Cleavage, in «Comparative Political Studies», n. 2, pp. 210-238.

Koole, R.A. (1994), The Vulnerability of the Modern Cadre Party in the Netherlands, in Katz e Mair (1994), pp. 278-303.

Lane, J.E. e S.O. Ersson (1996), European Politics. An Introduction, London, Sage.

Leyton-Henry, Z. (a cura di) (1982), Conservative Politics in Western Europe, London, Macmillan.

Lijphart, A. (1988), Le democrazie contemporanee, Bologna, Il Mulino.

Ljunggren, S.B. (1988), Conservatorism in Norway and Sweden, in Girvin (1988), pp. 120-144.

Luther, K.R. (1988), The Freiheitliche Partei Österreichs: Protest Party or Governing Party?, in Kirchner (1988), pp. 213-251.

Mair, P. (1992), The Problem of Party System Change, in «Journal of Theoretical Politics», n. 3, pp. 251-276. 
Minkenberg, M. e R. Inglehart (1989), Observations on Cultural Change and Postmodernism, in Gibbins (a cura di), pp. 251-256.

Morgan, R. e S. Silvestri (1983), I partiti moderati e conservatori europei, Roma, Edizioni Studio Tesi.

Morlino, L. (1988), Dall'autoritarismo alla democrazia: Portogallo, Spagna e Grecia, in N. Tranfaglia e M. Firpo (a cura di), La storia: l'età contemporanea, vol. IX, Torino, Utet, pp. 761-788.

- (1992), Partiti e consolidamento democratico, in Calise (1992), pp. 243-274.

- (1995), Political Parties and Democratic Consolidation in Southern Europe, in R. Gunther, P.N. Diamandouros e H.J. Puhle (a cura di), The Politics of Democratic Consolidation. Southern Europe in Comparative Perspective, Baltimore-London, Johns Hopkins University Press, pp. 315-388.

- (1996), Crisis of Parties and Change of Party System in Italy, in «Party Politics», vol. 2, n. 1, pp. 5-30.

- (1998), Democracy between Consolidation and Crisis. Parties, Groups and Citizens in Southern Europe, Oxford, Oxford University Press.

Morlino, L. e F. Mattei (1992), Vecchio e nuovo autoritarismo nell'Europa mediterranea, in «Rivista Italiana di Scienza Politica», vol. 22, n. 1 , pp. 137-160.

Müller, W.C. e B. Steininger (1994), Party Organisation and Party Competitiveness: The Case of the Austrian People's Party, 19451992, in «European Journal of Political Research», vol. 24, n. 1, pp.1-30.

Panebianco, A. (1982), Modelli di partito, Bologna, Il Mulino.

- (1986), Superstizioni politologiche?, in «il Mulino», n. 2, pp. 179190.

Pappalardo, A. (1992), Il rigore socialista: vincolo o scelta?, in Calise (1992), pp. 147-167.

Pappas, S.T. (1994), New Democracy: The Logics of A Conservative Party's Organization, Department of Sociology Yale University, testo non pubblicato.

- (1996), Grand Designs, Narrow Choices: Conservatives and Democracy in Southern Europe, EUI Fiesole, testo non pubblicato.

Pasquino, G. (1997), Corso di scienza della politica, Bologna, Il Mulino. Pedersen, N.M. (1983), Changing Patterns of Electoral Volatility in European Party Systems, 1948-1977, in H. Daalder e P. Mair (a cura di), Western European Party Systems: Continuity and Change, London, Sage, pp. 29-66.

Peele, G. (1988), The Character of Modern British Conservatism, in Girvin (1988), pp. 13-34.

Raniolo, F. (1997), I partiti conservatori nell'Europa Occidentale. Andamenti elettorali, memberships e partecipazione al governo, Tesi di dottorato, Facoltà di Scienza Politiche «C. Alfieri», Firenze. 
- (1998), Mezzo secolo di fortune elettorali dei partiti moderati e conservatori in Europa occidentale (1945-1996), in «Quaderni dell'Osservatorio Elettorale», n. 37, in corso di stampa.

Rokkan, S. (1982), Cittadini, elezioni e partiti, Bologna, Il Mulino.

Rudd, C. (1988), The Belgian Liberal Parties: Economic Radicals and Social Conservatives, in Kirchner (1988), pp. 178-212.

Sartori, G. (1965), Partiti e sistemi di partito, Firenze, Editrice Universitaria.

- (1976), Parties and Party System: A Framework for Analysis, Cambridge, Cambridge University Press.

- (1982), Teoria dei partiti e caso italiano, Milano, SugarCo.

Sartori, G. e L. Morlino (a cura di) (1991), La comparazione nelle scienze sociali, Bologna, Il Mulino.

Schlesinger, A.J. (1985), The New American Political Party, in «American Political Science Review», vol. 79, n. 4, pp. 1152-1169.

Smith, G. (1988), Between Left and Right: The Ambivalence of European Liberalism, in Kirchner (1988), pp. 16-28.

Steinmo, S., K. Thelen e F. Longstreth (a cura di) (1992), Structuring Politics. Historical Institutionalism in Comparative Analiysis, Cambridge, Cambridge University Press.

Taggart, P. (1995), New Populist Parties in Western Europe, in «West European Politics», n. 1, pp. 34-51.

Thomas, A.H. (1988), Liberalism in Denmark: Agrarian, Radical, still Influential, in Kirchner (1988), pp. 279-303.

Vassallo, S. (1997), Aspetti politico-istituzionali e governo della finanza pubblica, tesi di dottorato, Facoltà di Scienze Politiche «C. Alfieri», Firenze.

Ware, A. (1996), Political Parties and Party Systems, Oxford, Oxford University Press.

Wellhofer, S.E. (1979), Strategies for Party Organization and Voter Mobilization: Britian, Norway, and Argentina, in «Comparative Political Studies», n. 2, pp. 169-204. 